\title{
Review of Strategies to Reduce Central Line-Associated Bloodstream Infection (CLABSI) and Catheter-Associated Urinary Tract Infection (CAUTI) in Adult ICUs
}

\author{
Payal K. Patel, MD, MPH ${ }^{1,2 *}$, Ashwin Gupta, MD¹,3, Valerie M. Vaughn, MD³, Jason D. Mann, MSA ${ }^{3}$, \\ Jessica M. Ameling, MPH${ }^{3}$, Jennifer Meddings, MD, MSc $\mathrm{C}^{1,3,4,5}$
}

\begin{abstract}
${ }^{1}$ Ann Arbor Veterans Affairs Medical Center, Ann Arbor, Michigan; ${ }^{2}$ Department of Internal Medicine, Division of Infectious Diseases, University of Michigan Medical School, Ann Arbor, Michigan; ${ }^{3}$ Department of Internal Medicine, Division of General Medicine, University of Michigan Medical School, Ann Arbor, Michigan; ${ }^{4}$ Department of Pediatrics and Communicable Diseases, Division of General Pediatrics, University of Michigan Medical School, Ann Arbor, Michigan; ${ }^{5}$ Veterans Affairs Center for Clinical Management Research, Ann Arbor Veterans Affairs Medical Center, Ann Arbor, Michigan.
\end{abstract}

Central line-associated bloodstream infection (CLABSI) and catheter-associated urinary tract infection (CAUTI) are costly and morbid. Despite evidence-based guidelines, Some intensive care units (ICUs) continue to have elevated infection rates. In October 2015, we performed a systematic search of the peer-reviewed literature within the PubMed and Cochrane databases for interventions to reduce CLABSI and/or CAUTI in adult ICUs and synthesized findings using a narrative review process.

The interventions were categorized using a conceptual model, with stages applicable to both CAUTI and CLABSI prevention: (stage 0) avoid catheter if possible, (stage 1) ensure aseptic placement, (stage 2) maintain awareness and proper care of catheters in place, and (stage 3) promptly remove unnecessary catheters. We also looked for effective components that the 5 most successful (by reduction in infection rates) studies of each infection shared. Interventions that addressed multiple stages within the conceptual model were common in these successful studies. Assuring compliance with infection prevention efforts via auditing and timely feedback were also common. Hospitalists with patient safety interests may find this review informative for formulating quality improvement interventions to reduce these infections. Journal of Hospital Medicine 2018;13:105-116. Published online first November 8, 2017. (C) 2018 Society of Hospital Medicine entral line-associated bloodstream infection (CLAB$\mathrm{SI}$ ) and catheter-associated urinary tract infection (CAUTI) are morbid and expensive healthcare-associated infections (HAls). ${ }^{1-8}$ While these HAls are prevalent in intensive care units (ICUs) and general wards, most of the research, prevention efforts, and financial penalties have been focused in the ICU. ${ }^{9,10}$ For hospitalists, who are taking a larger role in caring for the critically ill, ${ }^{11,12}$ it is optimal to understand best preventive practices.

There has been a national push to standardize procedures and products to prevent CLABSI and CAUTI. 2,13-16 CLABSI has transitioned from a common ICU complication to a "never event." Success has been reflected in the prevention of 25,000 CLABSIs over the last decade, translating to a $58 \%$ reduction in infections, with 6000 deaths prevented and $\$ 414$ million saved. ${ }^{2}$

\footnotetext{
*Address for correspondence and reprint requests: Payal K. Patel, MD, MPH, Infectious Diseases Section, Ann Arbor VA Medical Center (111-i), 2215 Fuller Road, Ann Arbor, MI 48105; Telephone: 734-845-3460; Fax: 734-845-3290, E-mail: payalkp@umich.edu

Additional Supporting Information may be found in the online version of this article.
}

Received: March 1, 2017; Revised: May 26, 2017; Accepted: June 19, 2017

2018 Society of Hospital Medicine DOI 10.12788/jhm.2856
CLABSI prevention principles have been applied to CAUTI prevention (ie, aseptic insertion, maintenance care, prompting removal) but with slower adoption ${ }^{17}$ and fewer dramatic CAUTI reductions, ${ }^{18}$ due in part to weaker recognition ${ }^{19}$ of CAUTI as a serious clinical event, despite its morbidity ${ }^{20}$ and cost. ${ }^{21}$

Despite recent improvements in preventing HAls, there is a marked variability in how hospitals perform in preventing these infections. ${ }^{22}$ To inform infection prevention strategies for a largescale implementation project funded by the Agency for Healthcare Research and Quality and focused on ICUs with persistently elevated CLABSI and/or CAUTI rates, ${ }^{23}$ we performed a systematic search of interventions to prevent CLABSI and CAUTI in the ICU setting. This evidence was synthesized to help units select and prioritize interventions to prevent these HAls.

\section{METHODS}

\section{Literature Search Strategy}

We performed a systematic search to identify CLABSI and CAUTI prevention studies and synthesized findings using a narrative review process. Using criteria developed and refined from seminal articles on the topic, ${ }^{10,14,24-34}$ we searched the PubMed and Cochrane databases from their inception to October of 2015 using Medical Subject Headings (MeSHs) for "central venous catheters," "CLABSI," "central line associated bloodstream infection," "catheter related bloodstream 
infection," "intravascular devices," "urinary catheterization," "urinary catheters," "urinary tract infections," "CAUTI," and "catheter associated urinary tract infections" and filtered for articles containing the MeSHs "intensive care unit" and "ICU." Supplemental Figure 1 details the search, yielding 102 studies for CLABSI and 28 studies for CAUTI, including 7 studies with CLABSI and CAUTI interventions.

\section{Eligibility Criteria Review \\ Study Design}

We included randomized and nonrandomized studies that implemented at least 1 intervention to prevent CLABSI or CAU$\mathrm{TI}$ in an adult ICU setting and reported the preintervention or control group data to compare with the postintervention data. We excluded general ward, outpatient/ambulatory, and neonatal/pediatric settings. Interventions to prevent CLABSI or CAUTI were included. We excluded interventions focused on diagnosis or treatment or those that lacked adequate description of the intervention for replication. Studies with interventions that are no longer standard of care in the United States (US) were excluded, as were studies not available in English.

\section{Outcomes}

Primary Outcomes for Central Vascular Catheter Infection

- CLABSI: A lab-confirmed bloodstream infection in a patient who has had a central line for at least 48 hours on the date of the development of the bloodstream infection and without another known source of infection. We included studies that reported CLABSIs per 1000 central line days or those that provided data to permit calculation of this ratio. This measure is similar to current National Healthcare Safety Network (NHSN) surveillance definitions.22

- Catheter-related bloodstream infection (CRBSI): A labconfirmed bloodstream infection attributed to an intravascular catheter by a quantitative culture of the catheter tip or by differences in growth between catheter and peripheral venipuncture blood culture specimens.35 This microbiologic definition of a central line bloodstream infection was often used prior to NHSN reporting, with rates provided as the number of CRBSIs per 1000 central line days.

\section{Primary Outcome for Urinary Catheter Infection}

- CAUTI: Urinary tract infection occurring in patients during or after the recent use of an indwelling urinary catheter. We included studies that reported CAUTIs per 1000 urinary catheter days or those that provided data to permit calculation of this ratio (similar to the current NHSN surveillance definitions).22 We excluded studies where CAUTI was defined as bacteriuria alone, without symptoms.

\section{Secondary Outcomes}

- Central line utilization ratio: The device utilization ratio (DUR) measure of central line use is calculated as central line days divided by patient days.

- Urinary catheter utilization ratio: The DUR measure of urinary catheter use is calculated as indwelling urinary catheter

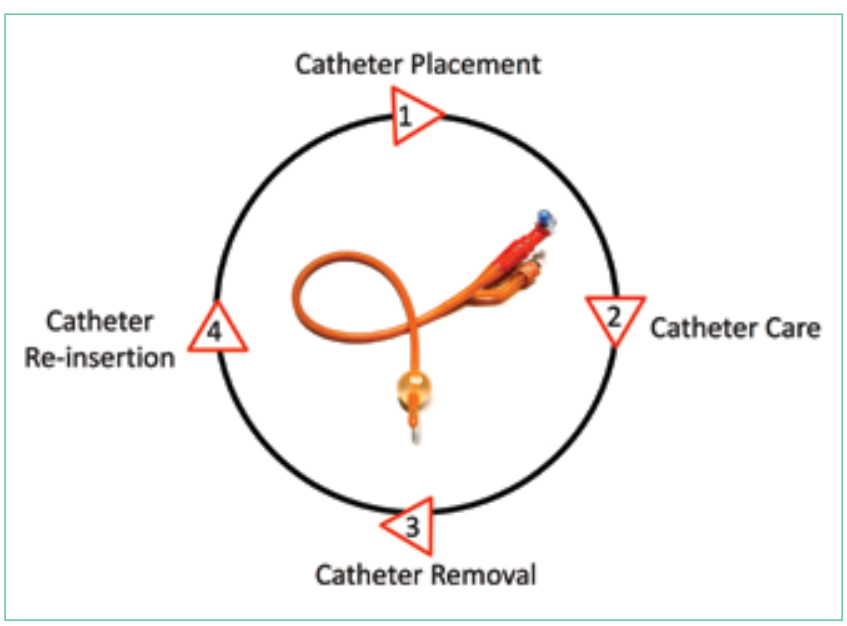

FIG 1. Life cycle of the urinary catheter.

From Meddings J, Saint S. Disrupting the life cycle of the urinary catheter. Clin Infect Dis. 2011;52(11):1291-1293, by permission of Clinical Infectious Disease.

days divided by patient days, as used in NHSN surveillance, excluding other catheter types.22 We excluded other measures of urinary catheter use because of a large variation in definitions, which limits the ability to compare measures across studies.

\section{Data Synthesis and Analysis}

Information on the ICU and intervention type, intervention components, outcomes, and whether interventions were in use prior to the study was abstracted by CAUTI and CLABSI experts (JM and PKP) and confirmed by a second author.

We compared interventions found in the literature to components of the previously published urinary catheter "life cycle," a conceptual model used to organize and prioritize interventions for a reduction in CAUTI (Figure 1). ${ }^{36}$ In this framework, there are 4 stages: (1) catheter placement, (2) catheter care, (3) catheter removal, and (4) catheter reinsertion. We sought to tailor the model for interventions in the ICU and for CLABSI prevention studies in addition to CAUTI prevention studies. In Table 1, we also provided the recommendation level for each intervention type provided in the CLABSI and CAUTI prevention guidelines from the Centers for Disease Control and Prevention Healthcare Infection Control Practices Advisory Committee, as close as was feasible, as the guidelines describe general strategies, not specific interventions. ${ }^{13,37}$

\section{RESULTS}

\section{Conceptual Model for Disrupting the Life Cycle} of a Catheter

Our data analysis demonstrated that components of the urinary catheter life cycle (Figure 1) were useful and could be applied to vascular catheters, but changes were needed to make the model more valuable to hospitalists implementing CLABSI and CAUTI prevention interventions. We found that the previously named stage 1 (catheter placement) is better described in 2 stages: stage 0 , avoid catheter if possible, and stage 1, ensure aseptic placement. Additionally, we tailored the model 
to include actionable language, describing ways to disrupt the life cycle. Finally, we added a component to represent interventions to improve implementation and sustainability, such as auditing compliance and timely feedback to clinicians. Thus, we introduce a new conceptual model, "Disrupting the Life Cycle of a Catheter" (Figure 2)-including stages appropriate for targeting both CAUTI and CLABSI prevention: (stage 0) avoid catheter if possible (ie, prevent catheter "life cycle" from beginning), (stage 1) ensure aseptic placement, (stage 2) optimize catheter maintenance care, and (stage 3) promptly remove unnecessary catheters-as well as apply interventions to improve implementation and sustainability. We used this modified conceptual model to synthesize the CLABSI and CAUTI prevention interventions found in the systematic search.

\section{Central Vascular Catheter Interventional Study Results \\ Characteristics of Included Central Vascular Catheter Infection Studies}

Of the 102 central vascular catheter (CVC) studies that met the inclusion criteria (reporting outcomes for 105 intervention cohorts), 59 studies $10,14,16,24-27,38-89$ reporting outcomes for 61 intervention cohorts were performed in the US. Study designs included 14 randomized controlled trials $(\mathrm{RCTs})^{48,64,68,74,79,90-98}$ and 88 before-after studies (Appendix Table 1). 10,14,16,24-27,33,3847.49-63,69-73,75-78,80-89,99-131 Many RCTs evaluated antimicrobial products (CVCs, hubs, bathing) as interventions, ${ }^{48,68,74,90-95,97,98}$ but a few RCTs studied interventions ${ }^{64,79,93}$ impacting catheter care or use (Appendix Table 1). Fifty-one studies took place in tertiary care hospitals and 55 in academic hospitals. Thirty-one studies were multicenter; the largest included 792 hospitals and 1071 ICUs. ${ }^{24}$ ICU bed size ranged from 5 to 59 .

\section{Central Vascular Catheter Study Outcomes}

Sixty-three studies reported CLABSI outcomes, and 39 reported CRBSI outcomes (Table 2). Many studies had preintervention or control rates above the 2013 NHSN 75th percentiles, ${ }^{22}$ which varied by ICU type. Preintervention or control infection rates per 1000 catheter days varied widely (means: CLABSI 7.5, CRBSI 6.3); US studies reported ranges of 1.1 to 12.1 CLABSI and 1.2 to 11.0 CRBSI per 1000 catheter days; non-US studies reported ranges of 1.4 to 45.9 CLABSI and 1.6 to 22.7 CRBSI per 1000 catheter days. Postintervention rates varied widely, with overall means of 2.8 CLABSI and 2.5 CRBSI per 1000 catheter days, including US study ranges of 0 to 8.9 CLABSI and 0 to $5.4 \mathrm{CRBSI}$, and non-US study ranges of 0 to $17.1 \mathrm{CLABSI}$ and 0 to 15.9 CRBSI.

Overall (Table 2), 99 of the 105 intervention cohorts described in the 102 studies reported either a reduced CLAB$\mathrm{SI}$ or a reduced CRBSI outcome, including all ICU types. Of the 63 CLABSI studies, 60 reported lower postintervention CLABSI rates, with a mean reduction of $62.6 \%$, though only 36 demonstrated statistical significance. Of the 39 studies that reported CRBSI outcomes, 37 reported lower postintervention CRBSI rates, with a mean reduction of $66 \%$, of which 23 were statistically significant.
Central line DURs were reported in only 5 studies; 3 reported decreased postintervention DURs (2 with statistical significance), with a mean $11.7 \%$ reduction (Table 2 ).

\section{Central Vascular Catheter Interventions}

CVC study interventions are summarized in Table 1, categorized by catheter life cycle component (Figure 2). Thirty-two included studies used a single intervention to prevent CVC infection. Interventions to avoid placement when possible were infrequent. Insertion-stage interventions were common and included avoiding the femoral site during placement, ensuring maximal sterile barriers, and chlorhexidine skin preparation. Standardizing basic products for central line insertion was often done by providing ICUs with a CLABSI insertion kit or stocked cart. In some studies, this was implemented prior to the intervention, and in others, the kit or cart itself was the intervention. Maintenance-stage interventions included scrubbing the hub prior to use, replacing wet or soiled dressings, accessing the catheter with sterile devices, and performing aseptic dressing changes. A recent systematic review and meta-analysis of CVC infection prevention studies indicated that implementing care bundles and/or checklists appears to yield stronger risk reductions than interventions without these components. ${ }^{132}$ The most common catheter removal interventions were daily audits of line removal and CLABSI rounds focused on ongoing catheter necessity.

Common implementation and sustainability interventions included outcome surveillance, such as feedback on CLABSI, and socio-adaptive interventions to prompt improvements in patient safety culture. Process and outcome surveillance as interventions were implemented in about one-quarter of the studies reviewed (AppendixTable 1).

\section{CAUTI Interventional Study Results Characteristics of Included CAUTI Studies}

Of the 28 CAUTI studies that met the inclusion criteria (reporting outcomes for 30 intervention cohorts), 14 studies (reporting outcomes for 16 intervention cohorts) were performed in the US. ${ }^{28,34,53,66,68,133-141}$ Study designs included 2 RCTs (focused on urinary catheter avoidance or removal ${ }^{142}$ and chlorhexidine bathing $\left.{ }^{68}\right)$ and 26 nonrandomized, before-after studies $28,30,33,34,53,66,109,114-116,133-141,143-149$ (Appendix Table 1). The number of hospitals per study varied from 1 to 53 , with the majority being single-hospital interventions.

\section{CAUTI Study Outcomes}

All 28 studies reported CAUTIs per 1000 catheter days for both intervention and comparison groups (Table 2). Preintervention or control CAUTI rates varied widely, with an overall mean of 12.5 CAUTIs per 1000 catheter days; US studies reported a range from 1.4 to 15.8 CAUTIs per 1000 catheter days; nonUS studies reported a range from 0.8 to 90.1 CAUTIs per 1000 catheter days. Many studies had preintervention or control rates above the 2013 NHSN 75th percentiles. ${ }^{22}$ Postintervention CAUTI rates varied widely, with an overall mean of 7.0 CAUTIs per 1000 catheter days, including a US study range 
TABLE 1. Intervention Characteristics of Included Studies (With HICPAC Recommendation Category ${ }^{\text {a) }}$

\section{A. Central Line-Associated Bloodstream Infection (CLABSI) Prevention Studies}

$\begin{array}{lc}\text { Stage of Life Cycle } & \text { b } \\ \text { Intervention Characteristic } & \mathrm{N}(\%) \text { of Included } \\ & \text { Studies Employing each } \\ \text { Intervention } \\ \text { Characteristic }(\mathrm{n}=102)\end{array}$

\section{Stage 0 Avoid $^{b}$}

Insertion Appropriateness (1A)

$3(2.9)$

Stage 1 Insertion ${ }^{b}$

\begin{tabular}{lc} 
Provide a Checklist (1B) & $25(24.5)$ \\
Avoid Femoral Site (1A) & $31(30.4)$ \\
Provide Insertion Kit/Cart (Not Graded) & $24(23.5)$ \\
Improve Hand Hygiene (1B) & $31(30.4)$ \\
Ensure Maximum Sterile Barriers (1B) & $36(35.3)$ \\
Chlorhexidine Skin Preparation (1A) & $30(29.4)$ \\
Cover Site with Sterile Dressing (1A) & $6(5.9)$ \\
Education on Insertion/Aseptic Technique (1A) & $55(53.9)$ \\
Empower Staff to Stop Procedure (Not Graded) & $10(9.8)$ \\
Process Surveillance (Not Graded) & $15(14.7)$ \\
Dedicated Staff for Catheter Insertion (1A) & $2(2.0)$ \\
\hline Stage 2 Maintenance & $5(4.9)$ \\
Scrub the Hub Prior to Use (1A) & $12(11.8)$ \\
Access Catheter with Sterile Device (1A) & $7(6.9)$ \\
Ensure Best Dressing Change Practices (1B) & $4(3.9)$ \\
Maintenance Checklist or Kit (1B) & $17(16.7)$ \\
\hline Chlorhexidine Bathing (II) & $5(4.9)$ \\
\hline
\end{tabular}

Stage 3 Removal $^{b}$

\begin{tabular}{lc} 
CLABSI Rounds or Daily Audit for Line Removal (1A) & $30(29.4)$ \\
Replacement of Emergent Lines (1B) & $4(3.9)$ \\
\hline Multiple Stages $^{b}$ & $19(18.6)$ \\
Antimicrobial CVC (1A) (Stages 2 \& 3) & $5(4.9)$ \\
Electronic Checklist for Documentation (Not Graded) (Stages \\
1-3)
\end{tabular}

Interventions to Improve Implementation \& Sustainability

Outcomes Surveillance: Feedback on CLABSI (Not Graded)

$27(26.5)$

Promote Culture of Quality and Safety (Not Graded)

$17(16.7)$

CUSP Implementation (1B)

\section{B. Catheter-Associated Urinary Tract Infection (CAUTI) Prevention Studie}

\begin{tabular}{lc}
\hline Stage of Life Cycle & $\mathrm{N}(\%)$ of Included \\
Intervention Characteristic & Studies Employing each \\
Intervention \\
Characteristic $(\mathrm{n}=28)$
\end{tabular}

Stage 0 Avoid $^{b}$

UC Placement Restrictions (1B)

$12(42.9)$

Promoting UC Alternatives (II)

$11(39.3)$

Require UC Order by Physicians (1B)

Stage 1 Insertion ${ }^{b}$

Standardized UC Kit (1B)

$3(10.7)$

Commercial Securement Device (1B)

$2(7.1)$

UC Insertion Cart (1B)

UC Aseptic Insertion Training (1B)

12 (42.9)

Stage 2 Maintenance ${ }^{b}$

$15(53.6)$

UTI Bundle Checklist in Rounds (1B)

$3(10.7)$

Bowel Management Program (Not Graded)

$2(7.1)$

Stage 3 Removal $^{b}$

Foley Catheter Rounds (1B)

UC Removal Reminder (1B)

$12(42.9)$

UC Removal Stop Order (1B)

$4(14.3)$

Multiple Stages ${ }^{b}$

Antimicrobial UCs (1B) (Stages $1 \&$ 2)

$2(7.1)$

Interventions also in ED or OR (Not Graded) (Stages 0 \& 3)

$2(7.1)$

Chlorhexidine Intervention (No Recommendations) (Stages 1 \& 2)

Healthcare Worker Hand Hygiene (1B) (Stages 1-3)

$13(46.4)$

Interventions to Improve Implementation \& Sustainability

Multidisciplinary Prep Meeting (Not Graded)

Data Feedback to ICUs (II)

$12(42.9)$

CAUTI Root Cause Analysis (Not Graded)

Measure Intervention Compliance (II)

Patient Education about UCs (Not Graded)

Adaptable Interventions, Reboot (Not Graded)

aHealthcare Infection Control Practices Advisory Committee (HICPAC) Recommendation Category from the Guidelines for Prevention of Intravascular Catheter-Related Infections, 2011, and Guideline for Prevention of Catheter-Associated Urinary Tract Infections, 2009: Category $1 \mathrm{~A}=$ strong recommendation supported by high to moderate quality evidence; Category $1 \mathrm{~B}=$ strong recommendation supported by low quality evidence; Category $1 \mathrm{C}=$ required by state or federal regulations; Category II = weak recommendation; No recommendation = unresolved issue; Not graded = not addressed in guidelines.

'Stages as defined in Figure 2 "Disrupting the Life Cycle of a Catheter."

NOTE: Abbreviations: CUSP, Comprehensive Unit-Based Safety Program; CVC, central vascular catheter; ED, emergency department; HICPAC, Healthcare Infection Control Practices Advisory Committee; ICU, intensive care unit; Int., intervention; OR, operating room; UC, urinary catheter, indwelling; UTI, urinary tract infection. 
TABLE 2. Study Outcomes, As Reported in Included ICU Intervention Studies

A. Central Line-Associated Bloodstream Infection (CLABSI) and Catheter-Related Bloodstream Infection (CRBSI)

\begin{tabular}{|c|c|c|c|c|c|c|}
\hline \multirow[b]{2}{*}{ Study } & \multicolumn{2}{|c|}{$\begin{array}{l}\text { CLABSIs per } 1000 \\
\text { Catheter Days }\end{array}$} & \multicolumn{2}{|c|}{$\begin{array}{l}\text { CRBSIs per } 1000 \\
\text { Catheter Days }\end{array}$} & \multicolumn{2}{|c|}{$\begin{array}{c}\text { Central Line } \\
\text { Utilization Rates }\end{array}$} \\
\hline & Pre-Int./Control Rate & Post-Int. Rate & Pre-Int./Control Rate & Post-Int. Rate & Pre-Int./Control Rate & Post-Int. Rate \\
\hline Allen $2014^{38}$ & $\begin{array}{l}2.72(\mathrm{M}) \\
1.09(\mathrm{~S})\end{array}$ & $\begin{array}{l}0.4(\mathrm{M})^{*} \\
1.14(\mathrm{~S})\end{array}$ & -- & -- & -- & -- \\
\hline Arora $2014^{39}$ & -- & -- & 3.5 & 1.6 & -- & -- \\
\hline Barsuk 200941 & 3.2 & $0.5^{*}$ & -- & -- & -- & -- \\
\hline Barsuk 201441 & 3.82 & $1.29^{*}$ & -- & -- & -- & -- \\
\hline Berenholtz $2014^{42}$ & 1.96 & $1.15^{*}$ & -- & -- & -- & -- \\
\hline Berenholtz $2004^{24}$ & 11.3 & 0 & -- & -- & -- & -- \\
\hline Bion $2013^{99}$ & 3.7 & $1.48^{*}$ & -- & -- & -- & -- \\
\hline Bonne $2015^{43}$ & 2.7 & $1.4^{*}$ & -- & -- & -- & -- \\
\hline Borschel $2006^{44}$ & -- & -- & 8.2 & $5.4^{*}$ & -- & -- \\
\hline Brun-Buisson $2004^{90}$ & -- & -- & 5.2 & 2 & -- & -- \\
\hline Burden $2012^{45}$ & -- & -- & 6.47 & $2.44^{*}$ & -- & -- \\
\hline Carrasco $2004^{91}$ & -- & -- & 3.24 & 2.6 & -- & -- \\
\hline Cherifi $2013^{100}$ & -- & -- & 4 & $1.81^{*}$ & -- & -- \\
\hline Cherry $2011^{46}$ & -- & -- & 3.53 & $2.26^{*}$ & -- & -- \\
\hline Chua $2010^{47}$ & -- & -- & 4.08 & $0^{*}$ & -- & -- \\
\hline Collin $1999^{48}$ & -- & -- & 3.95 & 1.14 & -- & -- \\
\hline Coopersmith $2002^{49}$ & -- & -- & 10.8 & $3.7^{*}$ & -- & -- \\
\hline Coopersmith $2004^{50}$ & -- & -- & 3.4 & 2.8 & -- & -- \\
\hline Corral $2003^{92}$ & -- & -- & 2.8 & $0.8^{*}$ & -- & -- \\
\hline Depalo $2010^{27}$ & 3.73 & $0.97^{*}$ & -- & -- & -- & -- \\
\hline Dixon $2010^{51}$ & 12.07 & $3.17^{*}$ & -- & -- & -- & -- \\
\hline Entesari-Tatafi $2015^{101}$ & 2.2 & $0.5^{*}$ & -- & -- & 1.22 & $1.37^{+}$ \\
\hline Exline $2013^{52}$ & 2.65 & $0.53^{*}$ & -- & -- & -- & \\
\hline Fox $2015^{53}$ & 1.1 & 0.5 & -- & -- & -- & -- \\
\hline Frankel $2005^{54}$ & -- & -- & 11 & $1.7^{*}$ & -- & -- \\
\hline Galpern $2008^{55}$ & -- & -- & 5 & $0.9^{*}$ & -- & -- \\
\hline Gozu $2011^{56}$ & 6 & $0.8^{*}$ & -- & -- & -- & - \\
\hline Hagau $2009^{93}$ & -- & -- & 6.9 & 3.1 & -- & -- \\
\hline Hakko $2015^{102}$ & 12.5 & 0 & -- & -- & -- & 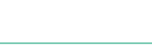 \\
\hline Hanna $2003^{57}$ & -- & -- & 1.4 & $0.46^{*}$ & -- & -- \\
\hline Hansen $2014^{103}$ & 1.4 & $0.46^{*}$ & -- & -- & -- & -- \\
\hline Hatler $2006^{58}$ & -- & -- & 12.8 & 2.88 & -- & -- \\
\hline Hermon $2015^{104}$ & 15.6 & 0.4 & -- & -- & & \\
\hline Hong $2013^{14}$ & 1.8 & 1.1 & -- & -- & -- & -- \\
\hline Jaggi $2013^{105}$ & 6.44 & 3.5 & -- & -- & -- & -- \\
\hline Jeong $2013^{107}$ & 4.7 & 1.8 & -- & -- & -- & -- \\
\hline Kalfon $2007^{94}$ & -- & -- & 1.9 & 2.1 & -- & -- \\
\hline Kamboj $2015^{59}$ & 4.93 & 4.47 & -- & -- & -- & -- \\
\hline Khalid $2013^{106}$ & 6.9 & $1.06^{*}$ & -- & -- & 0.65 & $0.51^{+}$ \\
\hline Khouli $2011^{60}$ & -- & -- & $\begin{array}{l}3.5(\mathrm{M}) \\
3.6(\mathrm{~S})\end{array}$ & $\begin{array}{l}1^{*}(\mathrm{M}) \\
3.4^{*}(\mathrm{~S})\end{array}$ & -- & -- \\
\hline Klintworth $2014^{108}$ & 2.3 & $0.9^{*}$ & -- & -- & -- & -- \\
\hline Koll $2008^{61}$ & 4.85 & $2.21^{*}$ & -- & -- & -- & -- \\
\hline Kurtz $2008^{95}$ & -- & -- & 11.4 & 4.8 & -- & -- \\
\hline
\end{tabular}


TABLE 2. Study Outcomes, As Reported in Included ICU Intervention Studies (continued)

A. Central Line-Associated Bloodstream Infection (CLABSI) and Catheter-Related Bloodstream Infection (CRBSI)

\section{CLABSIs per 1000 \\ Catheter Days}

\section{Study}

Latif $2015^{110}$

Leblebicioglu $2013^{109}$

Leon $2003^{97}$

Leon $2004^{98}$

Lin $2012^{25}$

Lin $2013^{16}$

Lobo $2010^{112}$

Longmate $2011^{111}$

Lopez $2011^{62}$

Lorente 2014 113

Maki $1997^{63}$

Marra $2010^{114}$

Marsteller $2012^{64}$

Martinez-Resendez $2014^{115}$

Mathur 2015'116

Mazi 2014 117

McMullan $2013^{65}$

Menegueti $2015^{118}$

Miller $2010^{66}$

Montecalvo $2012^{67}$

Mueller $2014^{87}$

Muto $2005^{10}$

Noto $2015^{68}$

Osma $2006^{96}$

Palomar $2013^{121}$

Paula $2012^{119}$

Peredo $2010^{122}$

Perez Parra 2010'23

Popovich $2009^{69}$

Popovich 201070

Pronovost 2006 26

Pronovost 201671

Rangachari $2015^{72}$

Reddy $2014^{120}$

Render $2011^{73}$

Rosenthal $2003^{124}$

Rosenthal $2010^{125}$

Rupp $2005^{74}$

Sacks $2014^{75}$

Salama $2016^{126}$

Salemi $2002^{76}$

Santana $2008^{127}$

Scheithauer 2014 128

Seguin $2010^{33}$

Shannon 200677

\begin{tabular}{cc}
\hline Pre-Int./Control Rate & Post-Int. Ra \\
\hline 3.02 & 0.74 \\
\hline 22.7 & $15.85^{*}$
\end{tabular}

$-$

1.5

1.49

$6.4 \quad 3.2^{\star}$

$2.56 \quad 0$

$14.37 \quad 15.23$

$27.6 \quad 7.2$

$3.87 \quad 1.5^{*}$

$6.37 \quad 0.76$

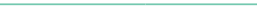

$\begin{array}{ll}-- & --\end{array}$

$\begin{array}{ll}8.53 & 2.23 \\ 6.4 & 2.9^{*}\end{array}$

$1.160 .8^{*}$

1.16
-

$--$

-- - -

$15.85 \quad 3.91$

$--$

$\begin{array}{ll}4.22 & 2.94\end{array}$

$7.7 \quad 1.4^{*}$

$2.5 \quad 0.76$

$--$

$2.99 \quad 1.47$

$3.85 \quad 1.8^{\star}$

$45.94 \quad 17.06^{*}$

16

$5.02 \quad 1.6^{*}$

$14.9 \quad 11.08$

$\begin{array}{ll}3.2 & 1.4 \\ 9.5 & 5.4^{*}\end{array}$

$5.87 \quad 1.51^{*}$

CRBSIs per 1000

Catheter Days

-- 22.7

\begin{tabular}{|c|c|c|c|}
\hline & \multicolumn{2}{|c|}{ Catheter Days } & Utilization Rates \\
\hline Rate & Pre-Int./Control Rate & Post-Int. Rate & Pre-Int./Control Rate \\
\hline . & -- & -- & -- \\
\hline $5^{*}$ & -- & -- & -- \\
\hline & 10.2 & 4.8 & -- \\
\hline & 22.7 & $15.85^{*}$ & -- \\
\hline & -- & -- & -- \\
\hline & -- & -- & -- \\
\hline & $12\left(\mathrm{M}^{1}\right)$ & $0\left(M^{1 *}\right)$ & -- \\
\hline & $16.2\left(M^{2}\right)$ & $13.7\left(\mathrm{M}^{2}\right)$ & \\
\hline & 3.4 & 0 & -- \\
\hline & -- & -- & -- \\
\hline & 5.04 & $0^{*}$ & -- \\
\hline & 7.6 & $1.6^{*}$ & -- \\
\hline & -- & -- & -- \\
\hline & -- & -- & -- \\
\hline 3 & -- & -- & -- \\
\hline & -- & -- & -- \\
\hline & -- & -- & -- \\
\hline & -- & -- & -- \\
\hline & 9.3 & $5.1^{*}$ & \\
\hline * & -- & -- & -- \\
\hline & -- & -- & -- \\
\hline & -- & -- & -- \\
\hline & 4.31 & $1.36^{*}$ & -- \\
\hline & -- & -- & -- \\
\hline & 1.6 & 5.3 & -- \\
\hline & 3.07 & $1.12^{*}$ & -- \\
\hline & -- & -- & -- \\
\hline & 6.7 & $2.4^{*}$ & -- \\
\hline & -- & -- & -- \\
\hline & 5.31 & 0.69 & -- \\
\hline & -- & -- & -- \\
\hline & -- & -- & -- \\
\hline & -- & -- & -- \\
\hline & 2.63 & 0.49 & -- \\
\hline * & -- & -- & -- \\
\hline & -- & -- & 0.32 \\
\hline $6^{*}$ & -- & -- & -- \\
\hline & -- & -- & -- \\
\hline & 1.24 & 0.42 & -- \\
\hline & -- & -- & -- \\
\hline 8 & -- & -- & -- \\
\hline
\end{tabular}

$22.7 \quad 15.85^{\star}$

$-$

$12\left(M^{\prime}\right)$

$16.2\left(M^{2}\right)$

$0\left(\mathrm{M}^{1 *}\right)$

\begin{tabular}{|c|c|c|c|}
\hline & \multicolumn{2}{|c|}{ Catheter Days } & Utilization Rates \\
\hline Rate & Pre-Int./Control Rate & Post-Int. Rate & Pre-Int./Control Rate \\
\hline . & -- & -- & -- \\
\hline $5^{*}$ & -- & -- & -- \\
\hline & 10.2 & 4.8 & -- \\
\hline & 22.7 & $15.85^{*}$ & -- \\
\hline & -- & -- & -- \\
\hline & -- & -- & -- \\
\hline & $12\left(\mathrm{M}^{1}\right)$ & $0\left(M^{1 *}\right)$ & -- \\
\hline & $16.2\left(M^{2}\right)$ & $13.7\left(\mathrm{M}^{2}\right)$ & \\
\hline & 3.4 & 0 & -- \\
\hline & -- & -- & -- \\
\hline & 5.04 & $0^{*}$ & -- \\
\hline & 7.6 & $1.6^{*}$ & -- \\
\hline & -- & -- & -- \\
\hline & -- & -- & -- \\
\hline 3 & -- & -- & -- \\
\hline & -- & -- & -- \\
\hline & -- & -- & -- \\
\hline & -- & -- & -- \\
\hline & 9.3 & $5.1^{*}$ & \\
\hline * & -- & -- & -- \\
\hline & -- & -- & -- \\
\hline & -- & -- & -- \\
\hline & 4.31 & $1.36^{*}$ & -- \\
\hline & -- & -- & -- \\
\hline & 1.6 & 5.3 & -- \\
\hline & 3.07 & $1.12^{*}$ & -- \\
\hline & -- & -- & -- \\
\hline & 6.7 & $2.4^{*}$ & -- \\
\hline & -- & -- & -- \\
\hline & 5.31 & 0.69 & -- \\
\hline & -- & -- & -- \\
\hline & -- & -- & -- \\
\hline & -- & -- & -- \\
\hline & 2.63 & 0.49 & -- \\
\hline * & -- & -- & -- \\
\hline & -- & -- & 0.32 \\
\hline $6^{*}$ & -- & -- & -- \\
\hline & -- & -- & -- \\
\hline & 1.24 & 0.42 & -- \\
\hline & -- & -- & -- \\
\hline 8 & -- & -- & -- \\
\hline
\end{tabular}

\begin{tabular}{|c|c|c|c|c|}
\hline \multicolumn{2}{|c|}{ Catheter Days } & \multicolumn{2}{|c|}{ Catheter Days } & Utilization Rates \\
\hline /Control Rate & Post-Int. Rate & Pre-Int./Control Rate & Post-Int. Rate & Pre-Int./Control Rate \\
\hline 3.02 & 0.74 & -- & -- & -- \\
\hline 22.7 & $15.85^{*}$ & -- & -- & -- \\
\hline-- & -- & 10.2 & 4.8 & -- \\
\hline-- & -- & 22.7 & $15.85^{*}$ & -- \\
\hline 1.5 & 0.6 & -- & -- & -- \\
\hline 1.49 & 0.25 & -- & -- & -- \\
\hline-- & -- & $12\left(M^{1}\right)$ & $0\left(M^{1 *}\right)$ & -- \\
\hline & & $16.2\left(M^{2}\right)$ & $13.7\left(M^{2}\right)$ & \\
\hline-- & -- & 3.4 & 0 & -- \\
\hline 5.7 & $0.2^{*}$ & -- & -- & -- \\
\hline-- & -- & 5.04 & $0^{*}$ & -- \\
\hline-- & -- & 7.6 & $1.6^{*}$ & -- \\
\hline 6.4 & $3.2^{*}$ & -- & -- & -- \\
\hline 2.56 & 0 & -- & -- & -- \\
\hline 14.37 & 15.23 & -- & -- & -- \\
\hline 27.6 & 7.2 & -- & -- & -- \\
\hline 3.87 & $1.5^{*}$ & -- & -- & -- \\
\hline 6.37 & 0.76 & -- & -- & -- \\
\hline-- & -- & 9.3 & $5.1^{*}$ & \\
\hline 8.53 & $2.23^{*}$ & -- & -- & -- \\
\hline 6.4 & $2.9^{*}$ & -- & -- & -- \\
\hline 1.16 & $0.8^{*}$ & -- & -- & -- \\
\hline-- & -- & 4.31 & $1.36^{*}$ & -- \\
\hline 5.45 & 5 & -- & -- & -- \\
\hline-- & -- & 1.6 & 5.3 & -- \\
\hline-- & -- & 3.07 & $1.12^{*}$ & -- \\
\hline 15.85 & 3.91 & -- & -- & -- \\
\hline-- & -- & 6.7 & $2.4^{*}$ & -- \\
\hline 4.22 & 2.94 & -- & -- & -- \\
\hline -- & -- & 5.31 & 0.69 & -- \\
\hline 3.81 & 4.6 & -- & -- & -- \\
\hline 7.7 & $1.4^{*}$ & -- & -- & -- \\
\hline 2.5 & 0.76 & -- & -- & -- \\
\hline-- & -- & 2.63 & 0.49 & -- \\
\hline 2.99 & $1.47^{\star}$ & -- & -- & -- \\
\hline 3.85 & $1.8^{*}$ & -- & -- & 0.32 \\
\hline 45.94 & $17.06^{*}$ & -- & -- & -- \\
\hline 16 & $7.4^{*}$ & -- & -- & -- \\
\hline-- & -- & 1.24 & 0.42 & -- \\
\hline 5.02 & $1.6^{*}$ & -- & -- & -- \\
\hline 14.9 & 11.08 & -- & -- & -- \\
\hline
\end{tabular}

\begin{tabular}{|c|c|c|c|}
\hline & \multicolumn{2}{|c|}{ Catheter Days } & Utilization Rates \\
\hline Rate & Pre-Int./Control Rate & Post-Int. Rate & Pre-Int./Control Rate \\
\hline . & -- & -- & -- \\
\hline $5^{*}$ & -- & -- & -- \\
\hline & 10.2 & 4.8 & -- \\
\hline & 22.7 & $15.85^{*}$ & -- \\
\hline & -- & -- & -- \\
\hline & -- & -- & -- \\
\hline & $12\left(\mathrm{M}^{1}\right)$ & $0\left(M^{1 *}\right)$ & -- \\
\hline & $16.2\left(M^{2}\right)$ & $13.7\left(\mathrm{M}^{2}\right)$ & \\
\hline & 3.4 & 0 & -- \\
\hline & -- & -- & -- \\
\hline & 5.04 & $0^{*}$ & -- \\
\hline & 7.6 & $1.6^{*}$ & -- \\
\hline & -- & -- & -- \\
\hline & -- & -- & -- \\
\hline 3 & -- & -- & -- \\
\hline & -- & -- & -- \\
\hline & -- & -- & -- \\
\hline & -- & -- & -- \\
\hline & 9.3 & $5.1^{*}$ & \\
\hline * & -- & -- & -- \\
\hline & -- & -- & -- \\
\hline & -- & -- & -- \\
\hline & 4.31 & $1.36^{*}$ & -- \\
\hline & -- & -- & -- \\
\hline & 1.6 & 5.3 & -- \\
\hline & 3.07 & $1.12^{*}$ & -- \\
\hline & -- & -- & -- \\
\hline & 6.7 & $2.4^{*}$ & -- \\
\hline & -- & -- & -- \\
\hline & 5.31 & 0.69 & -- \\
\hline & -- & -- & -- \\
\hline & -- & -- & -- \\
\hline & -- & -- & -- \\
\hline & 2.63 & 0.49 & -- \\
\hline * & -- & -- & -- \\
\hline & -- & -- & 0.32 \\
\hline $6^{*}$ & -- & -- & -- \\
\hline & -- & -- & -- \\
\hline & 1.24 & 0.42 & -- \\
\hline & -- & -- & -- \\
\hline 8 & -- & -- & -- \\
\hline
\end{tabular}

9.3

$5.1^{*}$

\begin{tabular}{|c|c|c|c|}
\hline & \multicolumn{2}{|c|}{ Catheter Days } & Utilization Rates \\
\hline Rate & Pre-Int./Control Rate & Post-Int. Rate & Pre-Int./Control Rate \\
\hline 4 & -- & -- & -- \\
\hline $5^{*}$ & -- & -- & -- \\
\hline & 10.2 & 4.8 & -- \\
\hline & 22.7 & $15.85^{*}$ & -- \\
\hline & -- & -- & -- \\
\hline 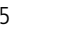 & -- & -- & -- \\
\hline & $12\left(\mathrm{M}^{1}\right)$ & $0\left(M^{1 *}\right)$ & -- \\
\hline & $16.2\left(M^{2}\right)$ & $13.7\left(M^{2}\right)$ & \\
\hline & 3.4 & 0 & -- \\
\hline & -- & -- & -- \\
\hline & 5.04 & $0^{*}$ & -- \\
\hline & 7.6 & $1.6^{*}$ & -- \\
\hline & -- & -- & -- \\
\hline & -- & -- & -- \\
\hline 23 & -- & -- & -- \\
\hline & -- & -- & -- \\
\hline & -- & -- & -- \\
\hline . & -- & -- & -- \\
\hline & 9.3 & $5.1^{*}$ & \\
\hline * & -- & -- & -- \\
\hline & -- & -- & -- \\
\hline & -- & -- & -- \\
\hline & 4.31 & $1.36^{*}$ & -- \\
\hline & -- & -- & -- \\
\hline & 1.6 & 5.3 & -- \\
\hline & 3.07 & $1.12^{*}$ & -- \\
\hline & -- & -- & -- \\
\hline & 6.7 & $2.4^{*}$ & -- \\
\hline 1 & -- & -- & -- \\
\hline & 5.31 & 0.69 & -- \\
\hline & -- & -- & -- \\
\hline & -- & -- & -- \\
\hline 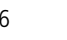 & -- & -- & -- \\
\hline & 2.63 & 0.49 & -- \\
\hline $7 *$ & -- & -- & -- \\
\hline & -- & -- & 0.32 \\
\hline $6^{*}$ & -- & -- & -- \\
\hline & -- & -- & -- \\
\hline & 1.24 & 0.42 & -- \\
\hline & -- & -- & -- \\
\hline 88 & -- & -- & -- \\
\hline
\end{tabular}

\begin{tabular}{|c|c|c|c|}
\hline & \multicolumn{2}{|c|}{ Catheter Days } & Utilization Rates \\
\hline Rate & Pre-Int./Control Rate & Post-Int. Rate & Pre-Int./Control Rate \\
\hline . & -- & -- & -- \\
\hline $5^{*}$ & -- & -- & -- \\
\hline & 10.2 & 4.8 & -- \\
\hline & 22.7 & $15.85^{*}$ & -- \\
\hline & -- & -- & -- \\
\hline & -- & -- & -- \\
\hline & $12\left(\mathrm{M}^{1}\right)$ & $0\left(M^{1 *}\right)$ & -- \\
\hline & $16.2\left(M^{2}\right)$ & $13.7\left(\mathrm{M}^{2}\right)$ & \\
\hline & 3.4 & 0 & -- \\
\hline & -- & -- & -- \\
\hline & 5.04 & $0^{*}$ & -- \\
\hline & 7.6 & $1.6^{*}$ & -- \\
\hline & -- & -- & -- \\
\hline & -- & -- & -- \\
\hline 3 & -- & -- & -- \\
\hline & -- & -- & -- \\
\hline & -- & -- & -- \\
\hline & -- & -- & -- \\
\hline & 9.3 & $5.1^{*}$ & \\
\hline * & -- & -- & -- \\
\hline & -- & -- & -- \\
\hline & -- & -- & -- \\
\hline & 4.31 & $1.36^{*}$ & -- \\
\hline & -- & -- & -- \\
\hline & 1.6 & 5.3 & -- \\
\hline & 3.07 & $1.12^{*}$ & -- \\
\hline & -- & -- & -- \\
\hline & 6.7 & $2.4^{*}$ & -- \\
\hline & -- & -- & -- \\
\hline & 5.31 & 0.69 & -- \\
\hline & -- & -- & -- \\
\hline & -- & -- & -- \\
\hline & -- & -- & -- \\
\hline & 2.63 & 0.49 & -- \\
\hline * & -- & -- & -- \\
\hline & -- & -- & 0.32 \\
\hline $6^{*}$ & -- & -- & -- \\
\hline & -- & -- & -- \\
\hline & 1.24 & 0.42 & -- \\
\hline & -- & -- & -- \\
\hline 8 & -- & -- & -- \\
\hline
\end{tabular}

\begin{tabular}{|c|c|c|c|}
\hline & \multicolumn{2}{|c|}{ Catheter Days } & Utilization Rates \\
\hline Rate & Pre-Int./Control Rate & Post-Int. Rate & Pre-Int./Control Rate \\
\hline 4 & -- & -- & -- \\
\hline $5^{*}$ & -- & -- & -- \\
\hline & 10.2 & 4.8 & -- \\
\hline & 22.7 & $15.85^{*}$ & -- \\
\hline & -- & -- & -- \\
\hline 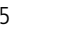 & -- & -- & -- \\
\hline & $12\left(\mathrm{M}^{1}\right)$ & $0\left(M^{1 *}\right)$ & -- \\
\hline & $16.2\left(M^{2}\right)$ & $13.7\left(M^{2}\right)$ & \\
\hline & 3.4 & 0 & -- \\
\hline & -- & -- & -- \\
\hline & 5.04 & $0^{*}$ & -- \\
\hline & 7.6 & $1.6^{*}$ & -- \\
\hline & -- & -- & -- \\
\hline & -- & -- & -- \\
\hline 23 & -- & -- & -- \\
\hline & -- & -- & -- \\
\hline & -- & -- & -- \\
\hline . & -- & -- & -- \\
\hline & 9.3 & $5.1^{*}$ & \\
\hline * & -- & -- & -- \\
\hline & -- & -- & -- \\
\hline & -- & -- & -- \\
\hline & 4.31 & $1.36^{*}$ & -- \\
\hline & -- & -- & -- \\
\hline & 1.6 & 5.3 & -- \\
\hline & 3.07 & $1.12^{*}$ & -- \\
\hline & -- & -- & -- \\
\hline & 6.7 & $2.4^{*}$ & -- \\
\hline 1 & -- & -- & -- \\
\hline & 5.31 & 0.69 & -- \\
\hline & -- & -- & -- \\
\hline & -- & -- & -- \\
\hline 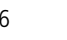 & -- & -- & -- \\
\hline & 2.63 & 0.49 & -- \\
\hline $7 *$ & -- & -- & -- \\
\hline & -- & -- & 0.32 \\
\hline $6^{*}$ & -- & -- & -- \\
\hline & -- & -- & -- \\
\hline & 1.24 & 0.42 & -- \\
\hline & -- & -- & -- \\
\hline 88 & -- & -- & -- \\
\hline
\end{tabular}

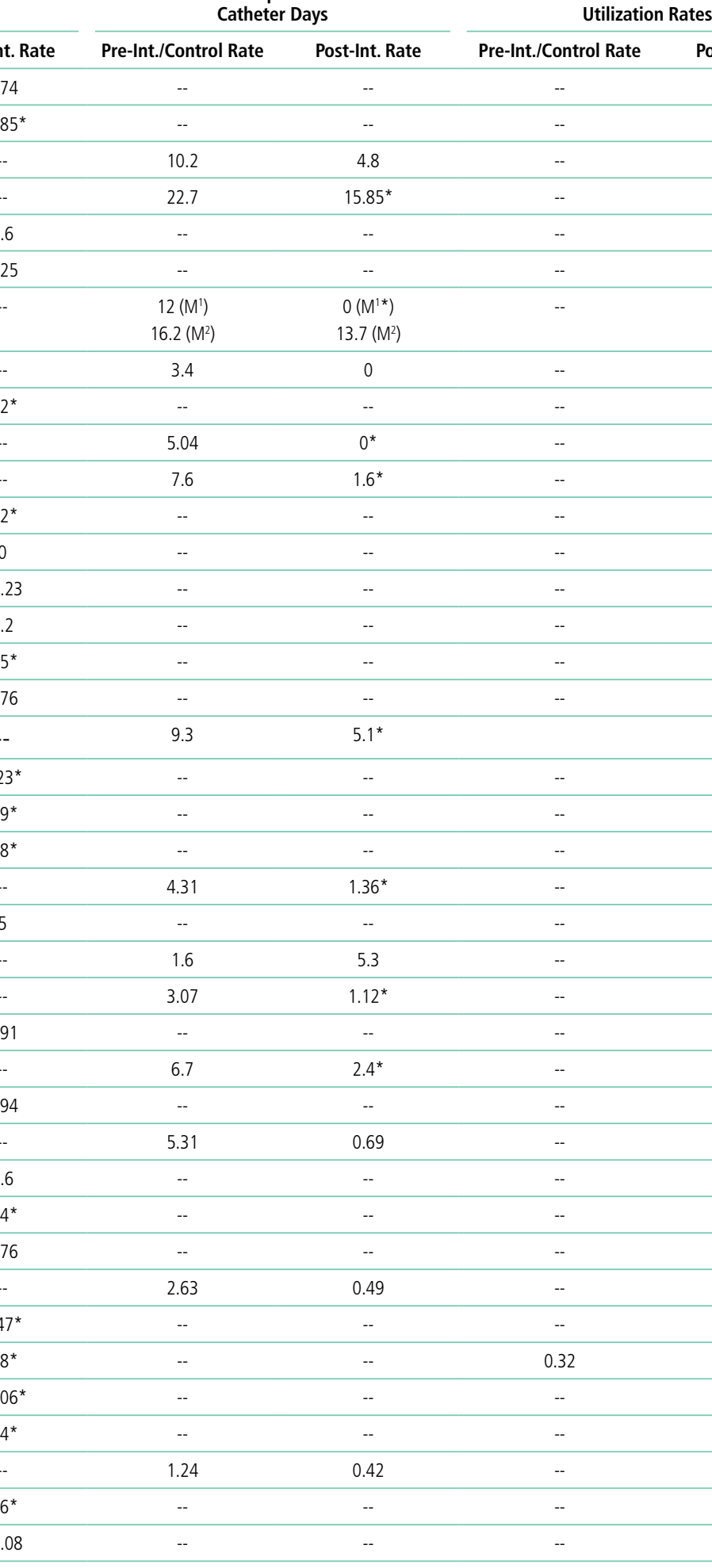

\begin{tabular}{|c|c|c|c|}
\hline & \multicolumn{2}{|c|}{ Catheter Days } & Utilization Rates \\
\hline Rate & Pre-Int./Control Rate & Post-Int. Rate & Pre-Int./Control Rate \\
\hline . & -- & -- & -- \\
\hline $5^{*}$ & -- & -- & -- \\
\hline & 10.2 & 4.8 & -- \\
\hline & 22.7 & $15.85^{*}$ & -- \\
\hline & -- & -- & -- \\
\hline & -- & -- & -- \\
\hline & $12\left(\mathrm{M}^{1}\right)$ & $0\left(M^{1 *}\right)$ & -- \\
\hline & $16.2\left(M^{2}\right)$ & $13.7\left(\mathrm{M}^{2}\right)$ & \\
\hline & 3.4 & 0 & -- \\
\hline & -- & -- & -- \\
\hline & 5.04 & $0^{*}$ & -- \\
\hline & 7.6 & $1.6^{*}$ & -- \\
\hline & -- & -- & -- \\
\hline & -- & -- & -- \\
\hline 3 & -- & -- & -- \\
\hline & -- & -- & -- \\
\hline & -- & -- & -- \\
\hline & -- & -- & -- \\
\hline & 9.3 & $5.1^{*}$ & \\
\hline * & -- & -- & -- \\
\hline & -- & -- & -- \\
\hline & -- & -- & -- \\
\hline & 4.31 & $1.36^{*}$ & -- \\
\hline & -- & -- & -- \\
\hline & 1.6 & 5.3 & -- \\
\hline & 3.07 & $1.12^{*}$ & -- \\
\hline & -- & -- & -- \\
\hline & 6.7 & $2.4^{*}$ & -- \\
\hline & -- & -- & -- \\
\hline & 5.31 & 0.69 & -- \\
\hline & -- & -- & -- \\
\hline & -- & -- & -- \\
\hline & -- & -- & -- \\
\hline & 2.63 & 0.49 & -- \\
\hline * & -- & -- & -- \\
\hline & -- & -- & 0.32 \\
\hline $6^{*}$ & -- & -- & -- \\
\hline & -- & -- & -- \\
\hline & 1.24 & 0.42 & -- \\
\hline & -- & -- & -- \\
\hline 8 & -- & -- & -- \\
\hline
\end{tabular}

\begin{tabular}{|c|c|c|c|}
\hline & \multicolumn{2}{|c|}{ Catheter Days } & Utilization Rates \\
\hline Rate & Pre-Int./Control Rate & Post-Int. Rate & Pre-Int./Control Rate \\
\hline . & -- & -- & -- \\
\hline $5^{*}$ & -- & -- & -- \\
\hline & 10.2 & 4.8 & -- \\
\hline & 22.7 & $15.85^{*}$ & -- \\
\hline & -- & -- & -- \\
\hline & -- & -- & -- \\
\hline & $12\left(\mathrm{M}^{1}\right)$ & $0\left(M^{1 *}\right)$ & -- \\
\hline & $16.2\left(M^{2}\right)$ & $13.7\left(\mathrm{M}^{2}\right)$ & \\
\hline & 3.4 & 0 & -- \\
\hline & -- & -- & -- \\
\hline & 5.04 & $0^{*}$ & -- \\
\hline & 7.6 & $1.6^{*}$ & -- \\
\hline & -- & -- & -- \\
\hline & -- & -- & -- \\
\hline 3 & -- & -- & -- \\
\hline & -- & -- & -- \\
\hline & -- & -- & -- \\
\hline & -- & -- & -- \\
\hline & 9.3 & $5.1^{*}$ & \\
\hline * & -- & -- & -- \\
\hline & -- & -- & -- \\
\hline & -- & -- & -- \\
\hline & 4.31 & $1.36^{*}$ & -- \\
\hline & -- & -- & -- \\
\hline & 1.6 & 5.3 & -- \\
\hline & 3.07 & $1.12^{*}$ & -- \\
\hline & -- & -- & -- \\
\hline & 6.7 & $2.4^{*}$ & -- \\
\hline & -- & -- & -- \\
\hline & 5.31 & 0.69 & -- \\
\hline & -- & -- & -- \\
\hline & -- & -- & -- \\
\hline & -- & -- & -- \\
\hline & 2.63 & 0.49 & -- \\
\hline * & -- & -- & -- \\
\hline & -- & -- & 0.32 \\
\hline $6^{*}$ & -- & -- & -- \\
\hline & -- & -- & -- \\
\hline & 1.24 & 0.42 & -- \\
\hline & -- & -- & -- \\
\hline 8 & -- & -- & -- \\
\hline
\end{tabular}

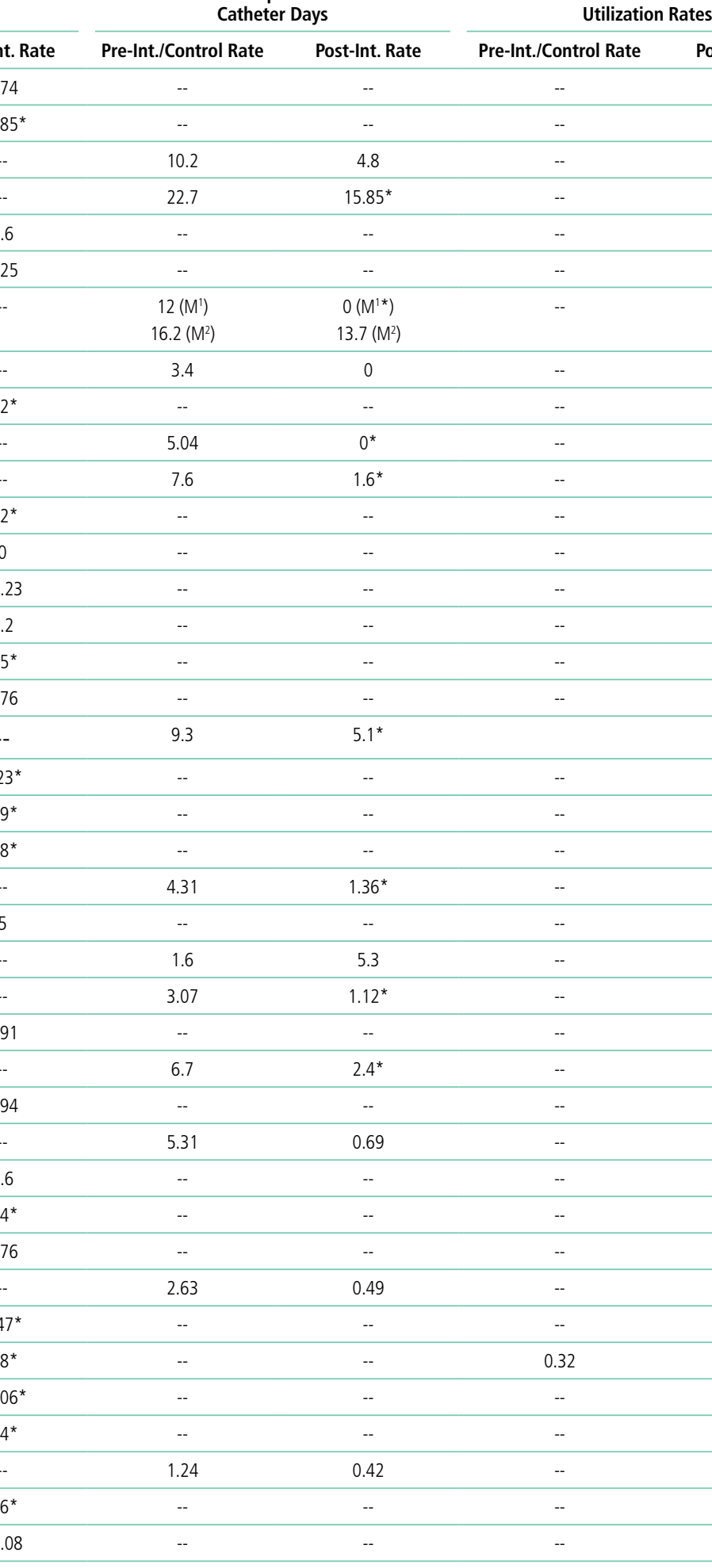

\begin{tabular}{|c|c|c|c|c|}
\hline \multicolumn{2}{|c|}{ Catheter Days } & \multicolumn{2}{|c|}{ Catheter Days } & Utilization Rates \\
\hline Control Rate & Post-Int. Rate & Pre-Int./Control Rate & Post-Int. Rate & Pre-Int./Control Rate \\
\hline 3.02 & 0.74 & -- & -- & -- \\
\hline 22.7 & $15.85^{\star}$ & -- & -- & -- \\
\hline-- & -- & 10.2 & 4.8 & -- \\
\hline -- & -- & 22.7 & $15.85^{*}$ & -- \\
\hline 1.5 & 0.6 & -- & -- & -- \\
\hline 1.49 & 0.25 & -- & -- & -- \\
\hline-- & -- & $12\left(M^{1}\right)$ & $0\left(M^{1 *}\right)$ & -- \\
\hline & & $16.2\left(M^{2}\right)$ & $13.7\left(M^{2}\right)$ & \\
\hline-- & -- & 3.4 & 0 & -- \\
\hline 5.7 & $0.2^{*}$ & -- & -- & -- \\
\hline-- & -- & 5.04 & $0^{*}$ & -- \\
\hline-- & -- & 7.6 & $1.6^{*}$ & -- \\
\hline 6.4 & $3.2^{*}$ & -- & -- & -- \\
\hline 2.56 & 0 & -- & -- & -- \\
\hline 14.37 & 15.23 & -- & -- & -- \\
\hline 27.6 & 7.2 & -- & -- & -- \\
\hline 3.87 & $1.5^{*}$ & -- & -- & -- \\
\hline 6.37 & 0.76 & -- & -- & -- \\
\hline-- & -- & 9.3 & $5.1^{*}$ & \\
\hline 8.53 & $2.23^{*}$ & -- & -- & -- \\
\hline 6.4 & $2.9^{*}$ & -- & -- & -- \\
\hline 1.16 & $0.8^{*}$ & -- & -- & -- \\
\hline -- & -- & 4.31 & $1.36^{*}$ & -- \\
\hline 5.45 & 5 & -- & -- & -- \\
\hline -- & -- & 1.6 & 5.3 & -- \\
\hline-- & -- & 3.07 & $1.12^{*}$ & -- \\
\hline 15.85 & 3.91 & -- & -- & -- \\
\hline-- & -- & 6.7 & $2.4^{*}$ & -- \\
\hline 4.22 & 2.94 & -- & -- & -- \\
\hline -- & -- & 5.31 & 0.69 & -- \\
\hline 3.81 & 4.6 & -- & -- & -- \\
\hline 7.7 & $1.4^{*}$ & -- & -- & -- \\
\hline 2.5 & 0.76 & -- & -- & -- \\
\hline -- & -- & 2.63 & 0.49 & -- \\
\hline 2.99 & $1.47^{*}$ & -- & -- & -- \\
\hline 3.85 & $1.8^{*}$ & -- & -- & 0.32 \\
\hline 45.94 & $17.06^{*}$ & -- & -- & -- \\
\hline 16 & $7.4^{*}$ & -- & -- & -- \\
\hline-- & -- & 1.24 & 0.42 & -- \\
\hline 5.02 & $1.6^{*}$ & -- & -- & -- \\
\hline 14.9 & 11.08 & -- & -- & -- \\
\hline
\end{tabular}

0.76

$7.4^{*}$

\begin{tabular}{|c|c|c|c|}
\hline & Cathete & & Utilizati \\
\hline nt. Rate & Pre-Int./Control Rate & Post-Int. Rate & Pre-Int./Control Rate \\
\hline .74 & -- & -- & -- \\
\hline $.85^{*}$ & -- & -- & -- \\
\hline-- & 10.2 & 4.8 & -- \\
\hline -- & 22.7 & $15.85^{*}$ & -- \\
\hline 0.6 & -- & -- & -- \\
\hline .25 & -- & -- & -- \\
\hline .- & $12\left(M^{1}\right)$ & $0\left(M^{1 *}\right)$ & -- \\
\hline & $16.2\left(M^{2}\right)$ & $13.7\left(M^{2}\right)$ & \\
\hline - & 3.4 & 0 & -- \\
\hline $.2^{*}$ & -- & -- & -- \\
\hline -- & 5.04 & $0^{*}$ & -- \\
\hline-- & 7.6 & $1.6^{*}$ & -- \\
\hline $.2^{*}$ & -- & -- & -- \\
\hline 0 & -- & -- & -- \\
\hline 5.23 & -- & -- & -- \\
\hline 7.2 & -- & -- & -- \\
\hline $.5^{*}$ & -- & -- & -- \\
\hline .76 & -- & -- & -- \\
\hline-- & 9.3 & $5.1^{*}$ & \\
\hline $23^{*}$ & -- & -- & -- \\
\hline $9^{*}$ & -- & -- & -- \\
\hline $8^{*}$ & -- & -- & -- \\
\hline -- & 4.31 & $1.36^{*}$ & -- \\
\hline 5 & -- & -- & -- \\
\hline-- & 1.6 & 5.3 & -- \\
\hline -- & 3.07 & $1.12^{*}$ & -- \\
\hline .91 & -- & -- & -- \\
\hline -- & 6.7 & $2.4^{*}$ & -- \\
\hline .94 & -- & -- & -- \\
\hline -- & 5.31 & 0.69 & -- \\
\hline 4.6 & -- & -- & -- \\
\hline $4^{*}$ & -- & -- & -- \\
\hline .76 & -- & -- & -- \\
\hline - & 2.63 & 0.49 & -- \\
\hline $47^{*}$ & -- & -- & -- \\
\hline $8^{*}$ & -- & -- & 0.32 \\
\hline $.06^{*}$ & -- & -- & -- \\
\hline $4^{*}$ & -- & -- & -- \\
\hline-- & 1.24 & 0.42 & -- \\
\hline $.6^{*}$ & -- & -- & -- \\
\hline 1.08 & -- & -- & -- \\
\hline
\end{tabular}

$--$

\begin{tabular}{|c|c|c|c|}
\hline & Cathet & & Utilizati \\
\hline t. Rate & Pre-Int./Control Rate & Post-Int. Rate & Pre-Int./Control Rate \\
\hline 74 & -- & -- & -- \\
\hline $85^{*}$ & -- & -- & -- \\
\hline & 10.2 & 4.8 & -- \\
\hline & 22.7 & $15.85^{*}$ & -- \\
\hline 6 & -- & -- & -- \\
\hline 25 & -- & -- & -- \\
\hline & $12\left(M^{1}\right)$ & $0\left(M^{1 *}\right)$ & -- \\
\hline & $16.2\left(M^{2}\right)$ & $13.7\left(M^{2}\right)$ & \\
\hline & 3.4 & 0 & -- \\
\hline $2^{*}$ & -- & -- & -- \\
\hline & 5.04 & $0^{*}$ & -- \\
\hline & 7.6 & $1.6^{*}$ & -- \\
\hline $2^{*}$ & -- & -- & -- \\
\hline & -- & -- & -- \\
\hline .23 & -- & -- & -- \\
\hline 2 & -- & -- & -- \\
\hline $5^{*}$ & -- & -- & -- \\
\hline 76 & -- & -- & -- \\
\hline & 9.3 & $5.1^{*}$ & \\
\hline 3 3* & -- & -- & -- \\
\hline $9^{*}$ & -- & -- & -- \\
\hline $8^{*}$ & -- & -- & -- \\
\hline & 4.31 & $1.36^{*}$ & -- \\
\hline & -- & -- & -- \\
\hline & 1.6 & 5.3 & -- \\
\hline & 3.07 & $1.12^{*}$ & -- \\
\hline 91 & -- & -- & -- \\
\hline & 6.7 & $2.4^{*}$ & -- \\
\hline 94 & -- & -- & -- \\
\hline & 5.31 & 0.69 & -- \\
\hline 6 & -- & -- & -- \\
\hline $4^{*}$ & -- & -- & -- \\
\hline 76 & -- & -- & -- \\
\hline & 2.63 & 0.49 & -- \\
\hline $17^{*}$ & -- & -- & -- \\
\hline $8^{*}$ & -- & -- & 0.32 \\
\hline $06^{*}$ & -- & -- & -- \\
\hline $4^{*}$ & -- & -- & -- \\
\hline & 1.24 & 0.42 & -- \\
\hline $6^{*}$ & -- & -- & -- \\
\hline .08 & -- & -- & -- \\
\hline
\end{tabular}

1.4

$.51^{*}$

$\begin{array}{cccc}-- & - & 2.8 & 0.7 \\ -- & -- & 10.6 & 0.39^{*}\end{array}$


TABLE 2. Study Outcomes, As Reported in Included ICU Intervention Studies (continued)

A. Central Line-Associated Bloodstream Infection (CLABSI) and Catheter-Related Bloodstream Infection (CRBSI)

\section{CLABSIs per 1000 \\ Catheter Days}

\section{Study}

Singh $2012^{129}$

Sopirala $2013^{78}$

Speroff $2011^{79}$

Thom $2014^{80}$

Venkatram $2010^{8}$

Vigorito $2011^{88}$

Wall $2005^{82}$

Walz $2015^{83}$

Warren $2004^{85}$

Warren $2006^{84}$

Watson $2009^{86}$

Zack $2008^{89}$

Zingg $2009^{131}$

Zingg 2014 $4^{130}$

$\begin{array}{cc}44 & 3.1^{*} \\ 3.35 & 0.28^{*} \\ 2.42 & 2.73 \\ 5 & 1.5^{*}\end{array}$

$\begin{array}{ll}- & 1.66\end{array}$

$7 \quad 3.8$

$5.86 \quad 0.33^{*}$

$9.4 \quad 5.5^{*}$

$11.28 .9^{*}$

$2.7 \quad 0$

$\begin{array}{ll}10.8 & 3.7\end{array}$

\begin{abstract}
Pre-Int./Control Rate
\end{abstract}

Post-Int. Rate

CRBSIs per 1000

Catheter Days

\section{Central Line \\ Utilization Rates}

\section{Pre-Int./Control Rate Post-Int. Rate}

Pre-Int./Control Rate

Post-Int. Rate

$1.5^{*}$

\begin{tabular}{cc}
-- & -- \\
-- & -- \\
-- & -- \\
\hline-- & -- \\
10.77 & $1.67^{*}$ \\
-- & -- \\
-- & -- \\
-- & -- \\
-- & -- \\
-- & -- \\
-- & -- \\
-- & -- \\
3.9 & $1^{*}$ \\
1.7 & $0.4^{*}$
\end{tabular}

\section{B. Catheter-Associated Urinary Tract Infection (CAUTI) Outcomes}

\section{CAUTIs per}

1000 Catheter Days

\section{Study}

Alexaitis $2014^{133}$

Amine $2014^{143}$

Apisarnthanarak $2007^{149}$

Chen $2013^{142}$

Dumigan $1998^{28}$

\section{Pre-Int./Control Rate}

3.85

90.12

23.4

17.2

$10.3(\mathrm{M})$
$15.8(\mathrm{~S})$

15.1 (C)

4.7

Elpern 2009 ${ }^{134}$

Fox $2015^{53}$

Fuchs $2011^{135}$

Huang 2004 ${ }^{30}$

Jain 2006 ${ }^{136}$

Kanj $2013^{144}$

Leblebicioglu $2013^{109}$

Marra, $2011^{150}$

Martinez-Resendez 2014 115

Mathur $2015^{116}$

Miller $2010^{66}$

Navoa-Ng $2013^{145}$

Noto $2015^{68}$

Popp, 2014 $4^{137}$

Reilly $2006^{138}$

Rosenthal $2012^{146}$

Saint, $2015^{139}$

Salama $2013^{147}$

Schelling $2015^{140}$

Seguin $2010^{33}$

Seyman 2014'148
9.1

2.88

11.5

3.8

13.07

10.63

7.6

16.68

37.13

7.48

11

1.54

2.7

$--$

7.86

1.4

5.5

8.18

5.0

0.87
Post-Int. Rate

3.06

65.69

$3.5^{*}$

10.3

8.6 (M)

$11.2(\mathrm{~S})$

$8.3^{*}(\mathrm{C})$
Urinary Catheter

Utilization Rates

$\begin{array}{ll}-- & - \\ -- & - \\ -- & - \\ -- & - \\ 0.44 & 0.4 \\ -- & - \\ -- & -- \\ -- & -- \\ -- & -- \\ 0.68 & 0.65+ \\ -- & -- \\ -- & - \\ -- & - \\ -- & --\end{array}$

\begin{tabular}{|c|c|c|}
\hline $0 *$ & -- & -- \\
\hline 5.6 & -- & -- \\
\hline 1.46 & -- & -- \\
\hline $8.3^{*}$ & -- & -- \\
\hline 2.4 & -- & -- \\
\hline $2.21^{*}$ & 0.96 & 0.96 \\
\hline $5.65^{*}$ & 0.9 & $0.84^{+}$ \\
\hline $5^{*}$ & 0.62 & 0.53 \\
\hline $12.62^{*}$ & -- & -- \\
\hline 15.5 & -- & -- \\
\hline $1.74^{*}$ & -- & -- \\
\hline $2.66^{*}$ & 0.67 & $0.6^{+}$ \\
\hline 1.09 & -- & -- \\
\hline 0 & -- & -- \\
\hline \% Reduction" & 0.96 & 0.86 \\
\hline 4.95 & 0.72 & 0.7 \\
\hline 2.1 & -- & -- \\
\hline 5.9 & -- & -- \\
\hline 0.93 & -- & -- \\
\hline 4.9 & -- & -- \\
\hline 1.88 & -- & -- \\
\hline
\end{tabular}


TABLE 2. Study Outcomes, As Reported in Included ICU Intervention Studies (continued)

\begin{tabular}{|c|c|c|c|c|}
\hline \multicolumn{5}{|c|}{ B. Catheter-Associated Urinary Tract Infection (CAUTI) Outcomes } \\
\hline \multirow[t]{2}{*}{ Study } & \multicolumn{2}{|c|}{$\begin{array}{c}\text { CAUTIs per } \\
1000 \text { Catheter Days }\end{array}$} & \multicolumn{2}{|c|}{$\begin{array}{l}\text { Urinary Catheter } \\
\text { Utilization Rates }\end{array}$} \\
\hline & Pre-Int./Control Rate & Post-Int. Rate & Pre-Int./Control Rate & Post-Int. Rate \\
\hline Sutherland $2015^{141}$ & 5.4 & 2.2 & .47 & .76 \\
\hline Titsworth $2012^{34}$ & 13.3 & $4^{*}$ & 1 & $0.73^{+}$ \\
\hline \multicolumn{5}{|c|}{ Superscript numbers denote multiple ICUs of the same type included in one study. } \\
\hline \multicolumn{5}{|c|}{$\begin{array}{l}\text { Statistically significant outcomes (when } \mathrm{p}<0.05 \text { or confidence interval did not contain a null hypothesis value) are noted with * for CLABSIs, CRBSIs, and CAUTIs and + for Central Line Device } \\
\text { and Urinary Catheter Utilization Rates. }\end{array}$} \\
\hline \multicolumn{5}{|c|}{$\begin{array}{l}\text { NOTE: Abbreviations: CLABSI,Central Line-Associated Bloodstream Infection; CRBSI,Catheter-Related Bloodstream Infection; CAUTI,Catheter-Associated Urinary Tract Infection; Int.,Interven- } \\
\text { tion; -- , not reported in study; ICU Types: M,Medical ICU, S,Surgical ICU, C,Cardiac ICU. }\end{array}$} \\
\hline
\end{tabular}

from 0 to 11.2 and a non-US study range from 1.9 to 65.7.

Overall (Table 2), 27 of the 30 intervention cohorts described in the 28 studies reported fewer CAUTIs, including all ICU types. Lower postintervention CAUTI rates were reported in 25 studies, with a mean $49.4 \%$ reduction, including 11 statistically significant reductions; many studies did not report the level of statistical significance or described inadequate power to detect a significant change (Table 2).

Urinary catheter utilization rates were reported for 11 studies (Table 2). A decreased urinary catheter utilization rate was reported in 7 studies (4 with statistically signficiant reductions), with a mean $16 \%$ reduction (Table 2). Other outcomes included cost savings, the potential for unintended negative outcomes, and clinician compliance with intervention components. Positive cost savings were reported in 5 studies. 30,34,133,141,149

\section{CAUTI Interventions}

Of the 28 included CAUTI prevention studies, only 5 studied single interventions. Interventions were categorized in Table 1 by "life cycle" stages or as interventions to improve implementation and sustainability (Figure 2). Interventions to restrict indwelling urinary catheter use were common, including creating lists of approved indications selected by unit or hospital policy and requiring catheter orders with approved indications. Eight studies published approved indication lists. 28,34,133-135,138,142,146 $\mathrm{Al}$ though several studies describe the encouragement and use of bladder scanners and urinary catheter alternatives, none described purchasing these catheter alternatives.

Interventions to avoid indwelling urinary catheters included education about external catheters, $28,34,109,133,140,14-146$ urinary retention protocols, ${ }^{34,144,135,141}$ and bladder scanner simulation training..$^{133}$ Interventions to improve aseptic insertion $28,34,66,109,116,139-141-143-146,150$ and maintenance care ${ }^{28,34,66,109,16,133,135,136,139-14,143-146,150}$ of urinary catheters were common. Four studies used a standardized urinary catheter kit or cart, 28,34,139,142 and 2 studies used a commercial urinary catheter securement device. ${ }^{34,140} \mathrm{~A}$ CAUTI bundle checklist in daily patient care rounds was tested in 3 studies (Table 1).66,136,150 Reminder and stop order strategies, with the potential to reduce CAUTI rates by $>50 \%$, 151 were included in 15 studies, with inteventions such as nurse-empowered stop orders. Several implementation and sustainability interventions were described, including socio-adaptive strategies such as holding multidisciplinary meetings to obtain unit or clinician feedback to inform design and improve buy-in and providing frequent feedback to ICU clinicians, including audits of catheter use appropriateness and catheter-associated infections.

\section{DISCUSSION}

This extensive literature review yielded a large body of literature demonstrating success in preventing CLABSI and CAUTI in all types of adult ICUs, including in general medical and surgical ICUs and in specialized units with historically higher rates, such as trauma, burn, and neurosurgical. Reported reductions in catheter infections were impressive ( $>65 \%$ for CLABSI or CRBSI and nearly 50\% for CAUTI), though several studies had limited power to detect statistical significance. DURs were reported more rarely (particularly for vascular catheters) and often without power to detect statistical significance. Nevertheless, 7 studies reported reduced urinary catheter use (16\% mean reduction), which would be anticipated to be clinically significant.

The conceptual model introduced for "Disrupting the Life Cycle of a Catheter" (Figure 2) can be a helpful tool for hospitalists and intensivists to assess and prioritize potential strategies for reducing catheter-associated infections. This study's results in-

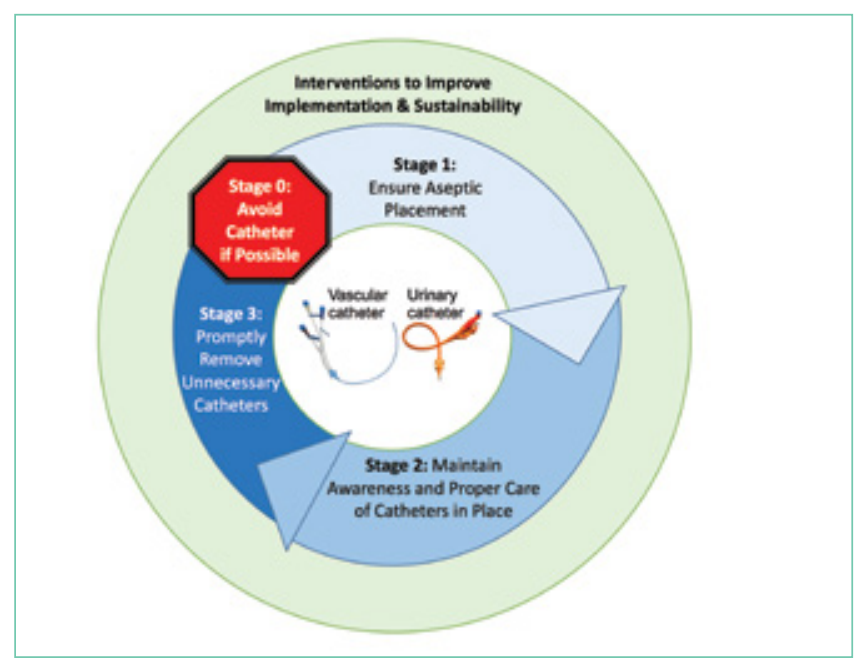

FIG 2. Disrupting the life cycle of a catheter.

Adapted from Meddings J, Saint S. Disrupting the life cycle of the urinary catheter. Clin Infect Dis. 2011;52(11):1291-129. 
dicate that CLABSI prevention studies often used interventions that optimize best practices during aseptic insertion and maintenance, but few studies emphasized reducing inappropriate central line use. Conversely, CAUTI prevention often targeted avoiding placement and prompting the removal of urinary catheters, with fewer studies evaluating innovative products or technical skill advancement for aseptic insertion or maintenance, though educational interventions to standardize aseptic catheter use were common. Recently, recommendations for reducing the inappropriate use of urinary catheters and intravenous catheters, including scenarios common in ICUs, were developed by using the rigorous RAND/UCLA Appropriateness Method ${ }^{152,153}$; these resources may be helpful to hospitalists designing and implementing interventions to reduce catheter use.

In reviewing the US studies of 5 units demonstrating the greatest success in preventing CLABSI ${ }^{56,62,65,78,83}$ and CAUTI, ${ }^{28,34,66,134}$ several shared features emerged. Interventions that addressed multiple steps within the life cycle of a catheter (avoidance, insertion, maintenance, and removal) were common. Previous work has shown that assuring compliance in infection prevention efforts is a key to success, ${ }^{154}$ and in both CLABSI and CAUTI studies, auditing was included in these successful interventions. Specifically for CLABSI, the checklist, a central quality improvement tool, was frequently associated with success. Unique to CAUTI, engaging a multidisciplinary team including nurse leadership seemed critical to optimize implementation and sustainability efforts. In addition, a focus on stage 3 (removal), including protocols to remove by default, was associated with success in CAUTI studies.

Our review was limited by a frequent lack of reporting of statistical significance or by inadequate power to detect a significant change and great variety. The ability to compare the impact of specific interventions is limited because studies varied greatly with respect to the type of intervention, duration of data collection, and outcomes assessed. We also anticipate that successful interventions are more likely to be published than are trials without success. Strengths include the use of a rigorous search process and the inclusion and review of several types of interventions implemented in ICUs.

In conclusion, despite high catheter use in ICUs, the literature includes many successful interventions for the prevention of vascular and urinary catheter infections in multiple ICU types. This review indicates that targeting multiple steps within the life cycle of a catheter, particularly when combined with interventions to optimize implementation and sustainability, can improve success in reducing CLABSI and CAUTI in the ICU.

\section{Acknowledgments}

The authors thank all members of the National Project Team for the AHRO Safety Program for Intensive Care Units: Preventing CLABSI and CAUTI.

Disclosure: Agency for Healthcare Research and Quality (AHRQ) contract \#HHSP2332015000161/HHSP23337002T provided funding for this study. J.M.'s other research is funded by AHRQ (2R01HS018334-04), the NIH-LRP program, the VA National Center for Patient Safety, VA Ann Arbor Patient Safety Center of Inquiry, the Health Research and Educational Trust, American Hospital Association and the Centers for Disease Control and Prevention. The findings and conclusions in this report are those of the authors and do not necessarily represent those of the sponsor, the Agency for Healthcare Research and Quality, or the US Department of Veterans Affairs. All authors report no conflicts of interest relevant to this article.

\section{References}

1. National and state healthcare-associated infections progress report. Centers for Disease Control and Prevention website. http://www.cdc.gov/hai/ progress-report/. 2016. Accessed January 10, 2016.

2. Srinivasan $A$, Wise $M, B e l l M$, et al. Vital signs: central line-associated blood stream infections-United States, 2001, 2008, and 2009. MMWR Morb Mortal Wkly Rep. 2011;60(8):243-248

3. Abramczyk ML, Carvalho WB, Carvalho ES, Medeiros EA. Nosocomial infection in a pediatric intensive care unit in a developing country. Braz J Infect Dis. 2003; $7(6): 375-380$.

4. Saint S. Clinical and economic consequences of nosocomial catheter-related bacteriuria. Am J Infect Control. 2000;28(1):68-75.

5. Ziegler MJ, Pellegrini DC, Safdar N. Attributable mortality of central line associated bloodstream infection: systematic review and meta-analysis. Infection. 2015;43(1):29-36.

6. Siempos, II, Kopterides P, Tsangaris I, Dimopoulou I, Armaganidis AE. Impact of catheter-related bloodstream infections on the mortality of critically ill patients: a meta-analysis. Crit Care Med. 2009;37(7):2283-2289.

7. Zingg W, Sax H, Inan C, et al. Hospital-wide surveillance of catheter-related bloodstream infection: from the expected to the unexpected. $J$ Hosp Infect. 2009;73(1):41-46

8. Chant C, Smith OM, Marshall JC, Friedrich JO. Relationship of catheter-associated urinary tract infection to mortality and length of stay in critically ill patients: a systematic review and meta-analysis of observational studies. Crit Care Med. 2011;39(5):1167-1173.

9. Lee GM, Kleinman K, Soumerai SB, et al. Effect of nonpayment for preventable infections in US hospitals. N Engl J Med. 2012;367(15):1428-1437.

10. Muto C, Herbert C, Harrison E, Edwards JR, et al. Reduction in central line-associated bloodstream infections among patients in intensive care units - Pennsylvania, April 2001-March 2005. MMWR Morb Mortal Wkly Rep. 2005;54(40):1013-1016.

11. Heisler M. Hospitalists and intensivists: partners in caring for the critically ill--the time has come. J Hosp Med. 2010;5(1):1-3.

12. Siegal EM, Dressler DD, Dichter JR, Gorman MJ, Lipsett PA. Training a hospitalist workforce to address the intensivist shortage in American hospitals: a position paper from the Society of Hospital Medicine and the Society of Critical Care Medicine. J Hosp Med. 2012;7(5):359-364.

13. Gould CV, Umscheid CA, Agarwal RK, Kuntz G, Pegues DA. Guideline for Prevention of Catheter-Associated Urinary Tract Infections 2009. Healthcare Infection Control Practices Advisory Committee (HICPAC). Centers for Disease Control and Prevention website. https://www.cdc.gov/infectioncontrol/guidelines/CAUTI/index.html. 2009. Accessed May 26, 2017.

14. Hong AL, Sawyer MD, Shore A, et al. Decreasing central-line-associated bloodstream infections in Connecticut intensive care units. J Healthc Qual. 2013;35(5):78-87

15. Weaver SJ, Weeks K, Pham JC, Pronovost PJ. On the CUSP: Stop BSI: evaluating the relationship between central line-associated bloodstream infection rate and patient safety climate profile. Am J Infect Control. 2014;42(10 Suppl):S203-S208.

16. Lin DM, Weeks K, Holzmueller CG, Pronovost PJ, Pham JC. Maintaining and sustaining the On the CUSP: stop BSI model in Hawaii. Jt Comm J Qual Patient Saf. 2013;39(2):51-60.

17. Krein SL, Fowler KE, Ratz D, Meddings J, Saint S. Preventing device-associated infections in US hospitals: national surveys from 2005 to 2013. BMJ Qual Saf. 2015;24(6):385-392

18. Department of Health and Human Services Action Plan to Prevent Healthcare-Associated Infections. Current progress on meeting these targets reviewed in 2013. https://health.gov/hcq/prevent-hai.asp. Accessed October 28, 2016.

19. Krein SL, Kowalski CP, Harrod M, Forman J, Saint S. Barriers to reducing urinary catheter use: a qualitative assessment of a statewide initiative. JAMA Intern Med. 2013;173(10):881-886.

20. Nicolle LE. Catheter associated urinary tract infections. Antimicrob Resist Infect Control. 2014;3:23.

21. Kennedy EH, Greene MT, Saint S. Estimating hospital costs of catheter-associated urinary tract infection. J Hosp Med. 2013;8(9):519-522.

22. Dudeck MA, Edwards JR, Allen-Bridson K, et al. National Healthcare Safety Network Report, data summary for 2013, Device-associated Module. Am J Infect Control. 2015;43:206-221. 
23. AHRQ Safety Program for Intensive Care Units: Preventing CLABSI and CAUTI. Agency for Healthcare Research and Quality website. http://www. ahrq.gov/professionals/quality-patient-safety/hais/tools/preventing/index. html. 2017. Accessed August 24, 2017

24. Berenholtz SM, Lubomski LH, Weeks K, et al. Eliminating central line-associated bloodstream infections: a national patient safety imperative. Infect Control Hosp Epidemiol. 2014;35(1):56-62

25. Lin DM, Weeks K, Bauer L, et al. Eradicating central line-associated bloodstreaminfectionsstatewide:theHawaiiexperience.AmJMedQual.2012;27(2): 124-129.

26. Pronovost $P$, Needham D, Berenholtz $S$, et al. An intervention to decrease catheter-related bloodstream infections in the ICU. N Engl J Med. 2006;355(26) 2725-2732.

27. DePalo VA, McNicoll L, Cornell M, Rocha JM, Adams L, Pronovost PJ. The Rhode Island ICU collaborative: a model for reducing central line-associated bloodstream infection and ventilator-associated pneumonia statewide. Qual Saf Health Care. 2010;19(6):555-561.

28. Dumigan DG, Kohan CA, Reed CR, Jekel JF, Fikrig MK. Utilizing national nosocomial infection surveillance system data to improve urinary tract in fection rates in three intensive-care units. Clin Perform Qual Health Care. 1998;6(4):172-178

29. Eggimann P, Harbarth S, Constantin MN, Touveneau S, Chevrolet JC, Pittet D Impact of a prevention strategy targeted at vascular-access care on incidence of infections acquired in intensive care. Lancet. 2000;355(9218):1864-1868.

30. Huang WC, Wann SR, Lin SL, et al. Catheter-associated urinary tract infections in intensive care units can be reduced by prompting physicians to remove unnecessary catheters. Infect Control Hosp Epidemiol. 2004;25(11):974-978

31. McLaws ML, Burrell AR. Zero risk for central line-associated bloodstream infection: are we there yet? Crit Care Med. 2012;40(2):388-393.

32. Miller SE, Maragakis LL. Central line-associated bloodstream infection prevention. Curr Opin Infect Dis. 2012;25(4):412-422.

33. Seguin $P$, Laviolle $B$, Isslame $S$, Coué $A$, Mallédant $Y$. Effectiveness of simple daily sensitization of physicians to the duration of central venous and urinary tract catheterization. Intensive Care Med. 2010;36(7):1202-1206.

34. Titsworth WL, Hester J, Correia T, et al. Reduction of catheter-associated urinary tract infections among patients in a neurological intensive care unit: a single institution's success. J Neurosurg. 2012;116(4):911-920.

35. Bouza E, Muñoz P, López-Rodríguez J, et al. A needleless closed system device (CLAVE) protects from intravascular catheter tip and hub colonization: a prospective randomized study. J Hosp Infect. 2003;54(4):279-287.

36. Meddings J, Saint S. Disrupting the life cycle of the urinary catheter. Clin Infect Dis. 2011;52(11):1291-1293.

37. O'Grady NP, Alexander M, Burns L, et al. Guidelines for the Prevention of Intravascular Catheter-Related Infections 2011. Healthcare Infection Control Practices Advisory Committee (HICPAC). Centers for Disease Control and Prevention website. https://www.cdc.gov/infectioncontrol/guidelines/BSI/ index.html. 2011. Accessed May 26, 2017.

38. Allen GB, Miller V, Nicholas C, et al. A multitiered strategy of simulation training, kit consolidation, and electronic documentation is associated with a reduction in central line-associated bloodstream infections. Am J Infect Control. 2014:42(6):643-648

39. Arora N, Patel K, Engell CA, LaRosa JA. The effect of interdisciplinary team rounds on urinary catheter and central venous catheter days and rates of infection. Am J Med Qual. 2014;29(4):329-334

40. Barsuk JH, Cohen ER, Feinglass J, McGaghie WC, Wayne DB. Use of simulation-based education to reduce catheter-related bloodstream infections. Arch Intern Med. 2009:169(15):1420-1423.

41. Barsuk JH, Cohen ER, Potts S, et al. Dissemination of a simulation-based mastery learning intervention reduces central line-associated bloodstream infections. BMJ Qual Saf. 2014;23(9):749-756

42. Berenholtz SM, Pronovost PJ, Lipsett PA, et al. Eliminating catheter-related bloodstream infections in the intensive care unit. Crit Care Med. 2004;32(10):2014-2020.

43. Bonne S, Mazuski JE, Sona $\mathrm{C}$, et al. Effectiveness of minocycline and rifampin vs chlorhexidine and silver sulfadiazine-impregnated central venous catheters in preventing central line-associated bloodstream infection in a high-volume academic intensive care unit: a before and after trial. J Am Coll Surg. 2015;221(3):739-747.

44. Borschel DM, Chenoweth CE, Kaufman SR, et al. Are antiseptic-coated central venous catheters effective in a real-world setting? Am J Infect Control. 2006;34(6):388-393.

45. Burden AR, Torjman MC, Dy GE, et al. Prevention of central venous catheter-related bloodstream infections: is it time to add simulation training to the prevention bundle? J Clin Anesth. 2012;24(7):555-560.
46. Cherry RA, West CE, Hamilton MC, Rafferty CM, Hollenbeak CS, Caputo GM. Reduction of central venous catheter associated blood stream infections following implementation of a resident oversight and credentialing policy. Patient Saf Surg. 2011;5(1):15.

47. Chua C, Wisniewski T, Ramos A, Schlepp M, Fildes JJ, Kuhls DA. Multidisciplinary trauma intensive care unit checklist: impact on infection rates. J Trauma Nurs. 2010;17(3):163-166.

48. Collin GR. Decreasing catheter colonization through the use of an antiseptic-impregnated catheter: a continuous quality improvement project. Chest. 1999; 115(6):1632-1640.

49. Coopersmith CM, Rebmann TL, Zack JE, et al. Effect of an education program on decreasing catheter-related bloodstream infections in the surgical intensive care unit. Crit Care Med. 2002;30(1):59-64.

50. Coopersmith CM, Zack JE, Ward MR, et al. The impact of bedside behavior on catheter-related bacteremia in the intensive care unit. Arch Surg. 2004;139(2):131-136.

51. Dixon JM, Carver RL. Daily chlorohexidine gluconate bathing with impregnated cloths results in statistically significant reduction in central line-associated bloodstream infections. Am J Infect Control. 2010;38(10):817-821.

52. Exline MC, Ali NA, Zikri N, et al. Beyond the bundle--journey of a tertiary care medical intensive care unit to zero central line-associated bloodstream infections. Crit Care. 2013;17(2):R41.

53. Fox C, Wavra T, Drake DA, et al. Use of a patient hand hygiene protocol to reduce hospital-acquired infections and improve nurses' hand washing. Am J Crit Care. 2015;24(3):216-224.

54. Frankel HL, Crede WB, Topal JE, Roumanis SA, Devlin MW, Foley AB. Use of corporate Six Sigma performance-improvement strategies to reduce incidence of catheter-related bloodstream infections in a surgical ICU. J Am Coll Surg. 2005;201(3):349-358.

55. Galpern D, Guerrero A, Tu A, Fahoum B, Wise L. Effectiveness of a central line bundle campaign on line-associated infections in the intensive care unit. Surgery. 2008;144(4):492-495.

56. Gozu A, Clay C, Younus F. Hospital-wide reduction in central line-associated bloodstream infections: a tale of two small community hospitals. Infect Control Hosp Epidemiol. 2011;32(6):619-622.

57. Hanna HA, Raad II, Hackett B, et al. Antibiotic-impregnated catheters associated with significant decrease in nosocomial and multidrug-resistant bacteremias in critically ill patients. Chest. 2003;124(3):1030-1038.

58. Hatler CW, Mast D, Corderella J, et al. Using evidence and process improvement strategies to enhance healthcare outcomes for the critically ill: a pilot project. Am J Crit Care. 2006;15(6):549-555.

59. Kamboj M, Blair R, Bell N, et al. Use of disinfection cap to reduce central-line-associated bloodstream infection and blood culture contamination among hematology-oncology patients. Infect Control Hosp Epidemiol. 2015:36:1401-1408

60. Khouli H, Jahnes K, Shapiro J, et al. Performance of medical residents in sterile techniques during central vein catheterization: randomized trial of efficacy of simulation-based training. Chest. 2011;139(1):80-87.

61. Koll BS, Straub TA, Jalon HS, Block R, Heller KS, Ruiz RE. The CLABs collaborative: a regionwide effort to improve the quality of care in hospitals. Jt Comm J Qual Patient Saf. 2008;34(12):713-723.

62. Lopez AC. A quality improvement program combining maximal barrier precaution compliance monitoring and daily chlorhexidine gluconate baths resulting in decreased central line bloodstream infections. Dimens Crit Care Nurs. 2011;30(5):293-298

63. Maki DG, Stolz SM, Wheeler S, Mermel LA. Prevention of central venous catheter-related bloodstream infection by use of an antiseptic-impregnated catheter. A randomized, controlled trial. Ann Intern Med. 1997:127(4):257-266.

64. Marsteller JA, Sexton JB, Hsu YJ, et al. A multicenter, phased, cluster-randomized controlled trial to reduce central line-associated bloodstream infections in intensive care units. Crit Care Med. 2012;40(11):2933-2939.

65. McMullan C, Propper G, Schuhmacher C, et al. A multidisciplinary approach to reduce central line-associated bloodstream infections. Jt Comm J Qual Patient Saf. 2013;39(2):61-69.

66. Miller RS, Norris PR, Jenkins JM, et al. Systems initiatives reduce healthcare-associated infections: a study of 22,928 device days in a single trauma unit. J Trauma. 2010;68(1):23-31.

67. Montecalvo MA, McKenna D, Yarrish R, et al. Chlorhexidine bathing to reduce central venous catheter-associated bloodstream infection: impact and sustainability. Am J Med. 2012;125(5):505-511.

68. Noto MJ, Domenico HJ, Byrne DW, et al. Chlorhexidine bathing and health care-associated infections: a randomized clinical trial. JAMA. 2015;313(4): 369-378.

69. Popovich KJ, Hota B, Hayes R, Weinstein RA, Hayden MK. Effectiveness of routine patient cleansing with chlorhexidine gluconate for infection pre- 
vention in the medical intensive care unit. Infect Control Hosp Epidemiol. 2009;30(10):959-963.

70. Popovich KJ, Hota B, Hayes R, Weinstein RA, Hayden MK. Daily skin cleansing with chlorhexidine did not reduce the rate of central-line associated bloodstream infection in a surgical intensive care unit. Intensive Care Med. 2010;36(5):854-858.

71. Pronovost PJ, Watson SR, Goeschel CA, Hyzy RC, Berenholtz SM. Sustaining reductions in central line-associated bloodstream infections in Michigan intensive care units: A 10-year analysis. Am J Med Qual. 2016;31(3):197-202.

72. Rangachari $\mathrm{P}$, Madaio M, Rethemeyer RK, et al. Cumulative impact of periodic top-down communications on infection prevention practices and outcomes in two units. Health Care Manage Rev. 2015;40(4):324-336.

73. Render ML, Hasselbeck R, Freyberg RW, et al. Reduction of central line infections in Veterans Administration intensive care units: an observational cohort using a central infrastructure to support learning and improvement. BMJ Qual Saf. 2011;20(8):725-732

74. Rupp ME, Lisco SJ, Lipsett PA, et al. Effect of a second-generation venous catheter impregnated with chlorhexidine and silver sulfadiazine on centra catheter-related infections: a randomized, controlled trial. Ann Intern Med. 2005; 143(8):570-580.

75. Sacks GD, Diggs BS, Hadjizacharia P, Green D, Salim A, Malinoski DJ. Reducing the rate of catheter-associated bloodstream infections in a surgical intensive care unit using the Institute for Healthcare Improvement Central Line Bundle. Am J Surg. 2014;207(6):817-823.

76. Salemi C, Canola MT, Eck EK. Hand washing and physicians: how to get them together. Infect Control Hosp Epidemiol. 2002;23(1):32-35.

77. Shannon RP, Frndak D, Grunden N, et al. Using real-time problem solving to eliminate central line infections. Jt Comm J Qual Patient Saf. 2006;32(9): 479-487.

78. Sopirala MM, Smyer J, Fawley L, et al. Sustained reduction of central line-associated bloodstream infections in an intensive care unit using a top-down and bottom-up approach. Am J Infect Control. 2013;41(2):183-184.

79. Speroff T, Ely EW, Greevy R, et al. Quality improvement projects targeting health care-associated infections: comparing Virtual Collaborative and Toolkit approaches. J Hosp Med. 2011;6(5):271-278.

80. Thom KA, Li S, Custer M, et al. Successful implementation of a unit-based quality nurse to reduce central line-associated bloodstream infections. Am J Infect Control. 2014;42(2):139-143.

81. Venkatram S, Rachmale S, Kanna B. Study of device use adjusted rates in health care-associated infections after implementation of "bundles" in a closed-model medical intensive care unit. J Crit Care. 2010;25(1):174.e11174.e18.

82. Wall RJ, Ely EW, Elasy TA, et al. Using real time process measurements to reduce catheter related bloodstream infections in the intensive care unit. Qual Saf Health Care. 2005;14(4):295-302.

83. Walz JM, Ellison RT 3rd, Mack DA, et al. The bundle "plus": the effect of a multidisciplinary team approach to eradicate central line-associated bloodstream infections. Anesth Analg. 2015;120(4):868-876.

84. Warren DK, Cosgrove SE, Diekema DJ, et al. A multicenter intervention to prevent catheter-associated bloodstream infections. Infect Control Hosp Epidemiol. 2006;27(7):662-669.

85. Warren DK, Zack JE, Mayfield JL, et al. The effect of an education program on the incidence of central venous catheter-associated bloodstream infection in a medical ICU. Chest. 2004;126(5):1612-1618.

86. Watson SR, George C, Martin M, Bogan B, Goeschel C, Pronovost PJ. Preventing central line-associated bloodstream infections and improving safety culture: a statewide experience. Jt Comm J Qual Patient Saf. 2009;35(12): 593-597.

87. Mueller JT, Wright AJ, Fedraw LA, et al. Standardizing central line safety: lessons learned for physician leaders. Am J Med Qual. 2014;29(3):191-199.

88. Vigorito MC, McNicoll L, Adams L, Sexton B. Improving safety culture results in Rhode Island ICUs: lessons learned from the development of action-oriented plans. Jt Comm J Qual Patient Saf. 2011;37(11):509-514.

89. Zack J. Zeroing in on zero tolerance for central line-associated bacteremia Am J Infect Control. 2008;36(10):S176.e1-S176.e2.

90. Brun-Buisson C, Doyon F, Sollet JP, Cochard JF, Cohen Y, Nitenberg G. Prevention of intravascular catheter-related infection with newer chlorhexidine-silver sulfadiazine-coated catheters: a randomized controlled trial. Intensive Care Med. 2004;30(5):837-843

91. Carrasco MN, Bueno A, de las Cuevas C, et al. Evaluation of a triple-lumen central venous heparin-coated catheter versus a catheter coated with chlorhexidine and silver sulfadiazine in critically ill patients. Intensive Care Med. 2004;30(4):633-638

92. Corral L, Nolla-Salas M, Ibañez-Nolla J, et al. A prospective, randomized study in critically ill patients using the Oligon Vantex catheter. J Hosp Infect. 2003:55(3):212-219.
93. Hagau N, Studnicska D, Gavrus RL, Csipak G, Hagau R, Slavcovici AV. Central venous catheter colonization and catheter-related bloodstream infections in critically ill patients: a comparison between standard and silver-integrated catheters. Eur J Anaesthesiol. 2009;26(9):752-758.

94. Kalfon P, de Vaumas C, Samba D, et al. Comparison of silver-impregnated with standard multi-lumen central venous catheters in critically ill patients. Crit Care Med. 2007;35(4):1032-1039.

95. Kurtz P, Rosa P, Penna G, et al. Antibiotic coated catheter to decrease infection: pilot study. Rev Bras Ter Intensiva. 2008;20(2):160-164.

96. Osma S, Kahveci SF, Kaya FN, et al. Efficacy of antiseptic-impregnated catheters on catheter colonization and catheter-related bloodstream infections in patients in an intensive care unit. J Hosp Infect. 2006;62(2):156-162.

97. León C, Alvarez-Lerma F, Ruiz-Santana S, et al. Antiseptic chamber-containing hub reduces central venous catheter-related infection: a prospective, randomized study. Crit Care Med. 2003;31(5):1318-1324.

98. León C, Ruiz-Santana S, Rello J, et al. Benefits of minocycline and rifampin-impregnated central venous catheters. A prospective, randomized, double-blind, controlled, multicenter trial. Intensive Care Med. 2004;30(10):1891-1899

99. Bion J, Richardson A, Hibbert P, et al. 'Matching Michigan': a 2-year stepped interventional programme to minimise central venous catheter-blood stream infections in intensive care units in England. BMJ Qual Saf. 2013;22(2):110-123

100. Cherifi S, Gerard M, Arias S, Byl B. A multicenter quasi-experimental study: impact of a central line infection control program using auditing and performance feedback in five Belgian intensive care units. Antimicrob Resist Infect Control. 2013;2(1):33

101. Entesari-Tatafi D, Orford N, Bailey MJ, Chonghaile MN, Lamb-Jenkins J, Athan E. Effectiveness of a care bundle to reduce central line-associated bloodstream infections. Med J Aust. 2015;202(5):247-250.

102. Hakko E, Guvenc S, Karaman I, Cakmak A, Erdem T, Cakmakci M. Longterm sustainability of zero central-line associated bloodstream infections is possible with high compliance with care bundle elements. East Mediterr Health J. 2015;21(4):293-298.

103. Hansen S, Schwab F, Schneider S, Sohr D, Gastmeier P, Geffers C. Time-series analysis to observe the impact of a centrally organized educational intervention on the prevention of central-line-associated bloodstream infections in 32 German intensive care units. J Hosp Infect. 2014;87(4):220-226.

104. Hermon A, Pain T, Beckett P, et al. Improving compliance with central venous catheter care bundles using electronic records. Nurs Crit Care. 2015;20(4):196-203.

105. Jaggi N, Rodrigues C, Rosenthal VD, et al. Impact of an international nosocomial infection control consortium multidimensional approach on central line-associated bloodstream infection rates in adult intensive care units in eight cities in India. Int J Infect Dis. 2013;17(12):e1218-e1224.

106. Khalid I, Al Salmi H, Qushmaq I, Al Hroub M, Kadri M, Qabajah MR. Itemizing the bundle: achieving and maintaining "zero" central line-associated bloodstream infection for over a year in a tertiary care hospital in Saudi Arabia. Am J Infect Control. 2013;41(12):1209-1213.

107. Jeong IS, Park SM, Lee JM, Song JY, Lee SJ. Effect of central line bundle on central line-associated bloodstream infections in intensive care units. Am J Infect Control. 2013;41(8):710-716.

108. Klintworth G, Stafford J, O'Connor M, et al. Beyond the intensive care unit bundle: Implementation of a successful hospital-wide initiative to reduce central line-associated bloodstream infections. Am J Infect Control. 2014;42(6):685-687.

109. Leblebicioglu H, Ersoz G, Rosenthal VD, et al. Impact of a multidimensional infection control approach on catheter-associated urinary tract infection rates in adult intensive care units in 10 cities of Turkey: International Nosocomial Infection Control Consortium findings (INICC). Am J Infect Control. 2013;41(10):885-891.

110. Latif A, Kelly B, Edrees $\mathrm{H}$, et al. Implementing a multifaceted intervention to decrease central line-associated bloodstream infections in SEHA (Abu Dhabi Health Services Company) intensive care units: the Abu Dhabi experience. Infect Control Hosp Epidemiol. 2015;36(7):816-822.

111. Longmate $A G$, Ellis $K S$, Boyle $L$, et al. Elimination of central-venous-catheter-related bloodstream infections from the intensive care unit. BMJ Qual Saf. 2011;20(2):174-180

112. Lobo RD, Levin AS, Oliveira MS, et al. Evaluation of interventions to reduce catheter-associated bloodstream infection: continuous tailored education versus one basic lecture. Am J Infect Control. 2010;38(6):440-448.

113. Lorente $L$, Lecuona $M$, Jiménez $A$, et al. Chlorhexidine-silver sulfadiazine-impregnated venous catheters save costs. Am J Infect Control. 2014;42(3):321-324.

114. Marra AR, Cal RG, Durão MS, et al. Impact of a program to prevent central line-associated bloodstream infection in the zero tolerance era. Am J Infect Control. 2010;38(6):434-439. 
115. Martínez-Reséndez MF, Garza-González E, Mendoza-Olazaran S, et al Impact of daily chlorhexidine baths and hand hygiene compliance on nosocomial infection rates in critically ill patients. Am J Infect Control. 2014;42(7):713-717.

116. Mathur P, Tak V, Gunjiyal J, et al. Device-associated infections at a level-1 trauma centre of a developing nation: impact of automated surveillance, training and feedbacks. Indian J Med Microbiol. 2015;33(1):51-62.

117. Mazi W, Begum Z, Abdulla D, et al. Central line-associated bloodstream infection in a trauma intensive care unit: impact of implementation of Society for Healthcare Epidemiology of America/Infectious Diseases Society of America practice guidelines. Am J Infect Control. 2014;42(8):865-867.

118. Menegueti MG, Ardison KM, Bellissimo-Rodrigues F, et al. The impact of implementation of bundle to reduce catheter-related bloodstream infection rates. J Clin Med Res. 2015;7(11):857-861.

119. Paula AP, Oliveira PR, Miranda EP, et al. The long-term impact of a program to prevent central line-associated bloodstream infections in a surgical intensive care unit. Clinics (Sao Paulo). 2012;67(8):969-970.

120. Reddy KK, Samuel A, Smiley KA, Weber S, Hon H. Reducing central line-associated bloodstream infections in three ICUs at a tertiary care hospital in the United Arab Emirates. Jt Comm J Qual Patient Saf. 2014;40(12):559-561.

121. Palomar M, Álvarez-Lerma F, Riera A, et al. Impact of a national multimodal intervention to prevent catheter-related bloodstream infection in the ICU: the Spanish experience. Crit Care Med. 2013;41(10):2364-2372.

122. Peredo R, Sabatier C, Villagrá A, et al. Reduction in catheter-related bloodstream infections in critically ill patients through a multiple system intervention. Eur J Clin Microbiol Infect Dis. 2010;29(9):1173-1177.

123. Pérez Parra A, Cruz Menárguez M, Pérez Granda MJ, Tomey MJ, Padilla B, Bouza $E$. A simple educational intervention to decrease incidence of central line-associated bloodstream infection (CLABSI) in intensive care units with low baseline incidence of CLABSI. Infect Control Hosp Epidemiol. 2010;31(9):964-967.

124. Rosenthal VD, Guzman S, Pezzotto SM, Crnich CJ. Effect of an infection control program using education and performance feedback on rates of intravascular device-associated bloodstream infections in intensive care units in Argentina. Am J Infect Control. 2003;31(7):405-409.

125. Rosenthal VD, Maki DG, Rodrigues C, et al. Impact of International Nosocomial Infection Control Consortium (INICC) strategy on central line-associated bloodstream infection rates in the intensive care units of 15 developing countries. Infect Control Hosp Epidemiol. 2010;31(12):1264-1272.

126. Salama MF, Jamal W, Mousa HA, Rotimi V. Implementation of central venous catheter bundle in an intensive care unit in Kuwait: Effect on central line-associated bloodstream infections. J Infect Public Health. 2016;9(1): 34-41.

127. Santana SL, Furtado GH, Wey SB, Medeiros EA. Impact of an education program on the incidence of central line-associated bloodstream infection in 2 medical-surgical intensive care units in Brazil. Infect Control Hosp Epidemiol. 2008;29(12):1171-1173.

128. Scheithauer S, Lewalter K, Schröder J, et al. Reduction of central venous line-associated bloodstream infection rates by using a chlorhexidine-containing dressing. Infection. 2014;42(1):155-159.

129. Singh S, Kumar RK, Sundaram KR, et al. Improving outcomes and reducing costs by modular training in infection control in a resource-limited setting. Int J Qual Health Care. 2012;24(6):641-648.

130. Zingg W, Cartier V, Inan C, et al. Hospital-wide multidisciplinary, multimodal intervention programme to reduce central venous catheter-associated bloodstream infection. PLoS One. 2014;9(4):e93898.

131. Zingg W, Imhof A, Maggiorini M, Stocker R, Keller E, Ruef C. Impact of a prevention strategy targeting hand hygiene and catheter care on the incidence of catheter-related bloodstream infections. Crit Care Med. 2009;37(7): 2167-2173.

132. Blot K, Bergs J, Vogelaers D, Blot S, Vandijck D. Prevention of centra line-associated bloodstream infections through quality improvement interventions: a systematic review and meta-analysis. Clin Infect Dis. 2014;59(1): 96-105.

133. Alexaitis I, Broome B. Implementation of a nurse-driven protocol to prevent catheter-associated urinary tract infections. J Nurs Care Qual. 2014;29(3) 245-252.

134. Elpern EH, Killeen K, Ketchem A, Wiley A, Patel G, Lateef O. Reducing use of indwelling urinary catheters and associated urinary tract infections. Am J Crit Care. 2009;18(6):535-541.

135. Fuchs MA, Sexton DJ, Thornlow DK, Champagne MT. Evaluation of an evidence-based, nurse-driven checklist to prevent hospital-acquired cath- eter-associated urinary tract infections in intensive care units. J Nurs Care Qual. 2011;26(2):101-109.

136. Jain M, Miller L, Belt D, King D, Berwick DM. Decline in ICU adverse events, nosocomial infections and cost through a quality improvement initiative focusing on teamwork and culture change. Qual Saf Health Care. 2006; 15(4):235-239

137. Popp JA, Layon AJ, Nappo R, Richards WT, Mozingo DW. Hospital-acquired infections and thermally injured patients: chlorhexidine gluconate baths work. Am J Infect Control. 2014;42(2):129-132.

138. Reilly L, Sullivan P, Ninni S, Fochesto D, Williams K, Fetherman B. Reducing foley catheter device days in an intensive care unit: using the evidence to change practice. AACN Adv Crit Care. 2006;17(3):272-283.

139. Saint S, Fowler KE, Sermak K, et al. Introducing the No Preventable Harms campaign: creating the safest health care system in the world, starting with catheter-associated urinary tract infection prevention. Am J Infect Control. 2015;43(3):254-259

140. Schelling K, Palamone J, Thomas K, et al. Reducing catheter-associated urinary tract infections in a neuro-spine intensive care unit. Am J Infect Control. 2015;43(8):892-894

141. Sutherland T, Beloff J, McGrath C, et al. A single-center multidisciplinary initiative to reduce catheter-associated urinary tract infection rates: Quality and financial implications. Health Care Manag (Frederick). 2015;34(3):218-224.

142. Chen YY, Chi MM, Chen YC, Chan YJ, Chou SS, Wang FD. Using a criteria-based reminder to reduce use of indwelling urinary catheters and decrease urinary tract infections. Am J Crit Care. 2013;22(2):105-114.

143. Amine AE, Helal MO, Bakr WM. Evaluation of an intervention program to prevent hospital-acquired catheter-associated urinary tract infections in an ICU in a rural Egypt hospital. GMS Hyg Infect Control. 2014;9(2):Doc15.

144. Kanj SS, Zahreddine N, Rosenthal VD, Alamuddin L, Kanafani Z, Molaeb B. Impact of a multidimensional infection control approach on catheter-associated urinary tract infection rates in an adult intensive care unit in Lebanon: International Nosocomial Infection Control Consortium (INICC) findings. Int J Infect Dis. 2013;17(9):e686-e690

145. Navoa-Ng JA, Berba R, Rosenthal VD, et al. Impact of an International Nosocomial Infection Control Consortium multidimensional approach on catheter-associated urinary tract infections in adult intensive care units in the Philippines: International Nosocomial Infection Control Consortium (INICC) findings. J Infect Public Health. 2013;6(5):389-399.

146. Rosenthal VD, Todi SK, Álvarez-Moreno C, et al. Impact of a multidimensional infection control strategy on catheter-associated urinary tract infection rates in the adult intensive care units of 15 developing countries: findings of the International Nosocomial Infection Control Consortium (INICC). Infection. 2012:40(5):517-526.

147. Salama MF, Jamal WY, Mousa HA, Al-Abdulghani KA, Rotimi VO. The effect of hand hygiene compliance on hospital-acquired infections in an ICU setting in a Kuwaiti teaching hospital. J Infect Public Health. 2013;6(1):27-34.

148. Seyman D, Oztoprak N, Berk H, Kizilates F, Emek M. Weekly chlorhexidine douche: does it reduce healthcare-associated bloodstream infections? Scand J Infect Dis. 2014;46(10):697-703.

149. Apisarnthanarak A, Thongphubeth K, Sirinvaravong S, et al. Effectiveness of multifaceted hospitalwide quality improvement programs featuring an intervention to remove unnecessary urinary catheters at a tertiary care center in Thailand. Infect Control Hosp Epidemiol. 2007;28(7):791-798.

150. Marra AR, Sampaio Camargo TZ, Gonçalves P, et al. Preventing catheter-associated urinary tract infection in the zero-tolerance era. Am J Infect Control. 2011;39(10):817-822

151. Meddings J, Rogers MA, Krein SL, Fakih MG, Olmsted RN, Saint S. Reducing unnecessary urinary catheter use and other strategies to prevent catheter-associated urinary tractinfection: an integrative review. BMJ Qual Saf.2014;23(4): 277-289.

152. Chopra V, Flanders SA, Saint S, et al. The Michigan Appropriateness Guide for Intravenous Catheters (MAGIC): results from a multispecialty panel using the RAND/UCLA appropriateness method. Ann Intern Med. 2015;163(6 Suppl):S1-S40.

153. Meddings J, Saint S, Fowler KE, et al. The Ann Arbor Criteria for appropriate urinary catheter use in hospitalized medical patients: results obtained by using the RAND/UCLA appropriateness method. Ann Intern Med. 2015;162(9 Suppl):S1-S34

154. Furuya EY, Dick AW, Herzig CT, Pogorzelska-Maziarz M, Larson EL, Stone PW. Central Line-Associated Bloodstream Infection Reduction and Bundle Compliance in Intensive Care Units: A National Study. Infect Control Hosp Epidemiol. 2016;37(7):805-810. 